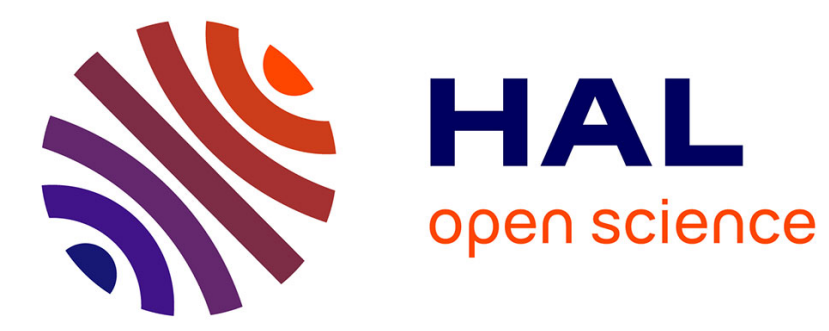

\title{
Modeling resistance to genetic control of insects
}

\author{
Nina Alphey, Michael B. Bonsall, Luke Alphey
}

\section{To cite this version:}

Nina Alphey, Michael B. Bonsall, Luke Alphey. Modeling resistance to genetic control of insects. Journal of Theoretical Biology, 2011, 270 (1), pp.42. 10.1016/j.jtbi.2010.11.016 . hal-00656339

\section{HAL Id: hal-00656339 \\ https://hal.science/hal-00656339}

Submitted on 4 Jan 2012

HAL is a multi-disciplinary open access archive for the deposit and dissemination of scientific research documents, whether they are published or not. The documents may come from teaching and research institutions in France or abroad, or from public or private research centers.
L'archive ouverte pluridisciplinaire HAL, est destinée au dépôt et à la diffusion de documents scientifiques de niveau recherche, publiés ou non, émanant des établissements d'enseignement et de recherche français ou étrangers, des laboratoires publics ou privés. 


\section{Author's Accepted Manuscript}

Modeling resistance to genetic control of insects

Nina Alphey, Michael B. Bonsall, Luke Alphey

PII: $\quad$ S0022-5193(10)00601-6

DOI: $\quad$ doi:10.1016/j.jtbi.2010.11.016

Reference: $\quad$ YJTBI6238

To appear in: $\quad$ Journal of Theoretical Biology

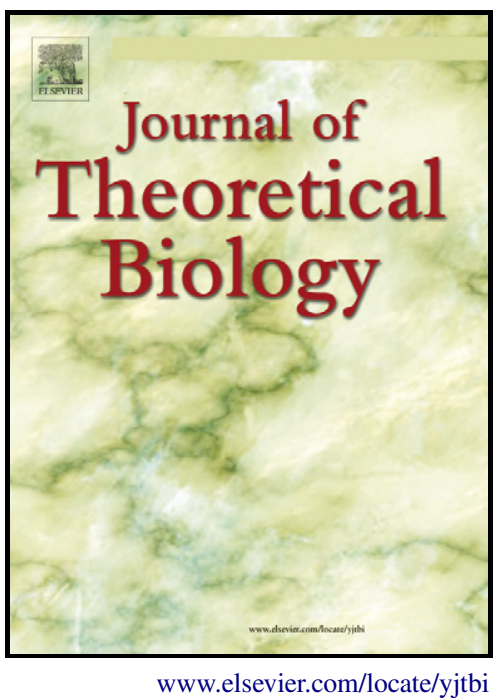

Received date: 19 March 2010

Revised date: $\quad 29$ September 2010

Accepted date: $\quad 9$ November 2010

Cite this article as: Nina Alphey, Michael B. Bonsall and Luke Alphey, Modeling resistance to genetic control of insects, Journal of Theoretical Biology, doi:10.1016/j.jtbi.2010.11.016

This is a PDF file of an unedited manuscript that has been accepted for publication. As a service to our customers we are providing this early version of the manuscript. The manuscript will undergo copyediting, typesetting, and review of the resulting galley proof before it is published in its final citable form. Please note that during the production process errors may be discovered which could affect the content, and all legal disclaimers that apply to the journal pertain. 


\section{Modeling resistance to genetic control of insects}

Nina Alphey a,b, ${ }^{\text {* }}$, Michael B. Bonsall ${ }^{\mathrm{a}}$ and Luke Alphey ${ }^{\mathrm{b}, \mathrm{c}}$

${ }^{a}$ Mathematical Ecology Research Group, Department of Zoology, University of Oxford, South Parks Road, Oxford OX1 3PS, United Kingdom

${ }^{\mathrm{b}}$ Oxitec Limited, 71 Milton Park, Oxford OX14 4RX, United Kingdom

${ }^{\mathrm{c}}$ Department of Zoology, University of Oxford, South Parks Road, Oxford OX1 3PS, United Kingdom

\section{*Corresponding author:}

Nina Alphey

Department of Zoology, University of Oxford, South Parks Road, Oxford OX1 3PS, United Kingdom

Tel: $+44(0) 1865281985$

Fax: +44(0)1865 310447

e-mail: nina.alphey@zoo.ox.ac.uk

Other author email addresses:

michael.bonsall@zoo.ox.ac.uk

luke.alphey@oxitec.com 


\section{ABSTRACT}

The sterile insect technique is an area-wide pest control method that reduces pest populations by releasing mass-reared sterile insects which compete for mates with wild insects. Modern molecular tools have created possibilities for improving and extending the sterile insect technique. As with any new insect control method, questions arise about potential resistance. Genetic RIDL ${ }^{\circledR}$ technology (Release of Insects carrying a Dominant Lethal) is a proposed modification of the technique, releasing insects that are homozygous for a repressible dominant lethal genetic construct rather than being sterilized by irradiation. Hypothetical resistance to the lethal mechanism is a potential threat to RIDL strategies' effectiveness. Using population genetic and population dynamic models, we assess the circumstances under which monogenic biochemically-based resistance could have a significant impact on the effectiveness of releases for population control. We assume that released insects would be homozygous susceptible to the lethal genetic construct and therefore releases would have a built-in element of resistance dilution. We find that this effect could prevent or limit the spread of resistance to RIDL constructs; the outcomes are subject to competing selective forces deriving from the fitness properties of resistance and the release ratio. Resistance that is spreading and capable of having a significant detrimental impact on population reduction is identifiable, signaling in advance a need for mitigating action.

FOOTNOTE $\quad$ RIDL $^{\circledR}$ is a registered trademark of Oxitec Limited, UK. 


\section{Key words/phrases:}

mathematical modeling

genetic control

RIDL

sterile insect technique (SIT)

resistance 


\section{Introduction}

Pest insects cause enormous harm to public health and economic damage to agriculture. Arthropods act as vectors for many human and veterinary diseases and annually destroy around $18 \%$ of the world crop production (Nicholson, 2007). The Sterile Insect Technique (SIT) is a pest population control method in which insects are mass-reared, sterilized by irradiation and released (Dyck et al., 2005; Knipling, 1955). Some wild insects mate with the released sterile insects and produce fewer progeny as a result. If sufficient insects are released for a sufficient period, populations of pest insects can be suppressed or perhaps eliminated locally. The SIT has been successful against a range of target species, mainly agricultural pests (Dyck et al., 2005).

Genetic technology, such as RIDL $^{\circledR}$ (Release of Insects carrying a Dominant Lethal), could modify the SIT by releasing insects that are not irradiated, but instead are homozygous for one or more dominant lethal genetic constructs that are repressible during mass-rearing (Alphey et al., 2007a; Alphey, 2007; Thomas et al., 2000). This approach is also known as autocidal biological control (Fryxell and Miller, 1995). Among the potential advantages claimed for genetic control over radiation-based SIT is the ability to engineer the timing or nature of the lethality. The preferred time of death varies between pests and contexts. Very early acting lethality would be preferable for pests where the larval stage causes the most damage; an embryonic lethal system has been developed in a model organism and work in a pest fruit fly is ongoing (Horn and Wimmer, 2003; Schetelig et al., 2007). Late-acting lethality that delays the time of death until after a density-dependent mortality phase, such as has been developed for RIDL technology in mosquitoes, can greatly enhance the SIT's 
effectiveness against a population that is limited by density-dependent processes (Atkinson et al., 2007; Phuc et al., 2007). Female-specific lethality would be advantageous for several reasons connected with facilitating separation of the sexes (Dyck et al., 2005). Release of only males, rather than both males and females, has been shown to make the SIT much more effective (Rendón et al., 2004). The efficiency of releases is improved because the released males have to seek out wild females; if both sexes were released there would be potential for assortative matings among the released sterile males and females and this would reduce the effect on control of the natural population. For insect vectors where females transmit disease, such as mosquitoes, release of females would be highly undesirable. However, in the absence of genetic sexing mechanisms, the biology of most species does not permit sex separation on the large scale required for the SIT and male-only releases are currently the exception rather than the norm.

A female-lethal version of RIDL, with insects homozygous for one or more female-specific dominant lethal genetic constructs, has been constructed in several species (Fu et al., 2007; Stainton et al., unpublished data). $F_{1}$ progeny of RIDL males and wild females inherit a dominant female-specific lethal gene; the $\mathrm{F}_{1}$ females die, thereby reducing the reproductive potential of the wild population, but the $\mathrm{F}_{1}$ males are viable and fertile. This provides a genetic sexing mechanism facilitating male only release, either by employing the femalelethal version of RIDL and withdrawing the repressor from the generation prior to release, or by combining a bisex-lethal system with female-lethality (with an independent means of repressing or inducing lethality) to permit male only release of bisex-lethal strains designed to kill progeny of both sexes in the field. 
Insects are well-known for developing resistance to insecticides. Insecticide resistance has been reported in most major insect disease vectors and against every class of chemical insecticide and insecticidal crops (Nicholson, 2007; Reynolds, 2007). "Behavioral resistance" to the SIT has been observed in isolated cases, where selection favors females that are able to identify and avoid mating with released sterile males (Dyck et al., 2005). As with any other new control method, resistance to the lethal mechanisms of the RIDL technology or other prospective methods of autocidal biological control is therefore a potential problem. None have so far been detected in any species at various stages of developing the technology (e.g. explicitly tested in Gong et al., 2005), but that does not preclude the possibility of resistance arising after the introduction of released insects, whether through new mutations or existing mutations in some wild populations that are capable of conferring resistance.

In practice, prior to commencing any release program there is no RIDL genetic construct in the wild, so there is no positive selection pressure and any resistance-associated allele would be expected to be at very low levels (especially if it impairs fitness and therefore carries a disadvantage that would tend to reduce its frequency) unless it also conferred some unrelated pleiotropic advantage. Evidence about resistance to other pest control methods generally supports this expectation. For example, from field data for several pests, if any resistance to insecticidal $\mathrm{Bt}$ crops could be detected at all, allele frequencies were of the order of $10^{-3}$ (Gould et al., 1997; Huang et al., 2007; Mahon et al., 2007; Stodola et al., 2006; Tabashnik et al., 2005). This was not surprising because the toxins were derived 
from a soil dwelling organism Bacillus thuringiensis and so there was some prior natural exposure. However, mutant alleles associated with resistance to malathion have been found in pinned specimens of Australian sheep blow fly Lucilia cuprina collected before the release of organophosphate insecticides (Hartley et al., 2006). This finding, in a small sample, suggests that the frequency of alleles that could confer resistance to a new product might be much higher than expected. If such polymorphisms are present at a high frequency before insecticide use, they are probably balanced polymorphisms (maintained by balancing selection such as heterozygote advantage) and therefore do not carry a clear net fitness cost in the absence of the new insecticide as would be expected for a new mutation (ffrench-Constant, 2007).

One possible measure to prevent or reverse the spread of resistance is to dilute the gene pool in the wild population with susceptible alleles. In radiation-based SIT, no viable hybrids are formed so there is no introgression of alleles from released insects into the wild population. With female-lethal RIDL, the $\mathrm{F}_{1}$ males are viable and fertile so alleles from the mass-reared strain could be spread into the wild population, with the female-lethal aspect still contributing to population suppression. Alleles from bisex-lethal RIDL strains might be pushed into the wild population if there were resistance to the RIDL genetic construct so that some progeny survive and reproduce.

In previous work, we explored insect resistance to crop plants engineered to produce insecticidal toxins (Bt crops), under arrangements where refuges of non-Bt plants are provided near insecticidal crops to provide a source of alleles conferring susceptibility to 
the toxins (Alphey et al., 2009; Alphey et al., 2007b). We proposed that control programs could combine Bt plants with the release of Bt-susceptible homozygote insects, either natural or engineered insects, to manage economically important agricultural pests. By providing an alternative source of susceptible alleles, release of homozygous susceptible female-lethal RIDL males (or wild type insects) is predicted to slow the spread of resistance to Bt and sufficient releases can prevent or reverse the spread of the resistant allele. Here we investigate the potential for introgression of alleles from released insects to act in a similar manner, to help manage biochemically-based genetic resistance to the autocidal genetic construct itself. This could apply to any form of biochemical resistance to the lethal effector molecule expressed by the transgenic construct, for example, through alteration of the target site or increased metabolism to clear the toxin.

The evolution of allele frequencies depends on selection pressure due to relative fitnesses of the different genotypes and properties of the resistance trait. Resistance to RIDL constructs has not yet been observed, so there is no data about its potential properties, and consequently we explore a wide range of theoretical values. There is limited data about the fitness penalties of the RIDL construct in the absence of resistance. Lethality achieved by a single copy of the construct in bisex-lethal or female-lethal strains can be $97-100 \%$ (Fu et al., 2007; Gong et al., 2005; Phuc et al., 2007). Some female-lethal strains have only a modest fitness penalty, if any, in males at 0-2\% lethality (Fu et al., 2007). Generally, from any transgenic insertion lines, only those with sufficiently close to $100 \%$ lethality in heterozygous form, and female-lethal strains with little or no fitness cost to heterozygous 
males, are chosen for further development. In such lines, lethality in homozygotes is $100 \%$ or very close (Fu et al., 2007; Stainton et al., unpublished data).

To some extent we can investigate the evolution of resistant allele frequency in isolation. However, to assess its impact on the main objective, which is to suppress or eliminate a population, we must explore the interaction between allele frequencies and population size, and to do this we must combine a population genetic framework with a population dynamic approach. For pests or disease vectors where both sexes are responsible for damage, the number of adults emerging at each generation is a proxy measure for the level of damage. For example, it is indicative of the level of crop damage by phytophagous agricultural pests, because every individual that survives to reproductive maturity has completed larval development on plants. For those disease vectors, such as mosquitoes, where only adult females transmit disease (when they take a blood meal), the number of adult females emerging at each generation is the relevant measure of potential harm. This might also be a good measure for some agricultural pests, such as tephritid fruit flies where oviposition damage is economically harmful.

Here, through mathematical modeling, we explore the potential for proposed release strategies to contribute to managing a hypothetical heritable biochemical resistance to RIDL genetic constructs. We examine the evolution of RIDL-resistant allele frequency and of the frequency of the RIDL construct, considering both dynamics over time and equilibria. We investigate the impact of the release ratio, initial resistant allele frequency and genetic properties of the resistant allele. First we consider dominant or recessive, 
completely effective resistance with no fitness costs and then gradually relax assumptions to allow the strength and fitness costs of resistance to vary across the whole range of possible values. We then explore the interaction between pest control and resistance management by modeling relative population size over time.

\section{Methods}

Parameter definitions and symbols are set out in Table 1.

\subsection{Population genetics model}

We use a deterministic, non-spatial, discrete-generation population genetic model of a closed homogeneous population, with random mating, and no immigration, emigration or mutation. We assume a 1:1 sex ratio.

The number of individuals surviving to reproductive maturity depends on the relative fitness values of the immature life stages. Embryonic, larval or pupal susceptibility to the lethal effect of a RIDL genetic construct is assumed to be controlled by a single autosomal locus with two alternative alleles: resistant $R$ (frequency $p$ in adults emerging in the wild) and susceptible $S$ (frequency $q, p+q=1$ ). There are three possible genotypes at this locus: $S S, S R$ and $R R$. For convenience, we use the terms "larvae" and "larval stage" to refer to all immature stages, although engineered lethality might in principle be designed to prevent egg hatch, pupation or adult eclosion instead of being fatal during larval development. 
The resistant allele may have fitness costs, which we assume also take effect during the larval stage. The relative fitness of immature individuals of genotype $i(i: S S, S R$ or $R R)$ is $\psi_{i}$, where fitness is measured relative to $S S$ homozygote wild type insects (so $\psi_{S S}=1$ ).

Fitness costs are not over- or under-dominant $\left(\psi_{R R} \leq \psi_{S R} \leq \psi_{S S}=1\right)$.

Engineered males for release ("RIDL males") carry a repressible dominant lethal genetic construct, for which they are homozygous. The RIDL construct is either bisex-lethal "BL" (where individuals of either sex inheriting one or more copies of the construct are nonviable in the absence of the repressor), or female-lethal "FL" (which similarly renders females non-viable but allows males to survive). There are two possible alleles at the insertion site of the RIDL construct: $L$, representing insertion of the construct (frequency $l$ in adults emerging in the wild), and the wild type allele $w$ (frequency $1-l$ ), representing absence of the construct, giving three possible genotypes at this locus: $w w, L w$ and $L L$. We assume there is no linkage between the RIDL insertion and the locus controlling resistance and neither of these is sex-linked.

The RIDL construct imposes a relative fitness cost $\varepsilon_{k}$ during the larval stage, where $k$ denotes either a "target" or "non-target" of the engineered lethality. The fitness penalty $\varepsilon_{\text {target }}$ is equal to 1 if the construct is fully lethal, and applies to both sexes for a bisex-lethal (BL) RIDL construct and to females only for a female-lethal (FL) construct. The sexspecific fitness cost $\varepsilon_{\text {non-target }}$ imposed by an FL construct on males, either takes a lower value, to allow for a modest associated fitness penalty (for example, due to insertional mutagenesis and/or expression of a marker gene incorporated into the construct (Marrelli et 
al., 2006)), or is zero (having no effect). We assume that the effect of the construct is multiplicative, so the relative fitness of genotype $j(j: w w, L w$ or $L L)$ in the absence of resistance is

$$
\Omega_{j}=\left(1-\varepsilon_{k}\right)^{\eta_{j}}
$$

where $\eta_{j}$ is the number of copies of the RIDL construct that the individual possesses ( 0 for $w w, 1$ for $L w$ or 2 for $L L)$.

We represent the resistance trait as reduced susceptibility to the RIDL construct, by scaling the fitness penalty $\varepsilon$ by a factor $\gamma_{i}(i: S S, S R$ or $R R)$. By definition of resistance, susceptible homozygotes are fully susceptible to the RIDL construct $\left(\gamma_{S S}=1\right)$ and $R R$ individuals are less susceptible than $S S\left(\gamma_{R R}<\gamma_{S S}\right)$. Resistance may be incomplete $\left(\gamma_{R R} \geq 0\right)$, meaning that resistance might only reduce the fitness penalty associated with the $L$ construct $\left(\gamma_{R R}>0\right)$, rather than negate its effect altogether (complete resistance, $\gamma_{R R}=0$ ). We assume that resistance is not over- or under- dominant $\left(\gamma_{R R} \leq \gamma_{S R} \leq \gamma_{S S}=1\right)$.

Combining all these features, the relative fitness of larvae of genotype $i, j, k(i: S S, S R$ or $R R, j: w w, L w$ or $L L, k$ : target or non-target) is then

$$
\Omega_{i j k}=\psi_{i}\left(1-\varepsilon_{k} \gamma_{i}\right)^{\eta_{j}}
$$

There are potentially 18 genotypes in this model: combinations of 3 variants at the $S / R$ locus, 3 variants at the $L / w$ locus, and (for FL only) 2 variants in target or non-target sex. 
No release. Our baseline case is where the $R$ allele is present but not at fixation $\left(0<p_{0}<1\right)$ in a naïve population (i.e., one with no $L$ alleles in the wild, $l_{0}=0$, which would be the case before any RIDL release program commenced) and no release strategy is being employed ("NR" no release). In this scenario, only the $S / R$ genotype is relevant (no $L$ allele is ever present as none are introduced) and relative fitness is $\Omega_{i j k}=\psi_{i}$ for all genotypes that can arise. Using standard population genetic theory (Gillespie, 1998) shows that the change in resistance allele frequency in a generation, $\Delta p$, is

$$
\begin{gathered}
\Delta p=\frac{p^{2} \Omega_{R R}+p q \Omega_{S R}}{q^{2} \Omega_{S S}+2 p q \Omega_{S R}+p^{2} \Omega_{R R}}-p \\
=\frac{p q\left(p\left(\psi_{R R}-\psi_{S R}\right)+q\left(\psi_{S R}-\psi_{S S}\right)\right)}{q^{2} \psi_{S S}+2 p q \psi_{S R}+p^{2} \psi_{R R}}
\end{gathered}
$$

Where $p, q \neq 0,1$ (resistance allele present but not at fixation), the sign of $\Delta p$ is determined by the sign of $p\left(\psi_{R R}-\psi_{S R}\right)+q\left(\psi_{S R}-\psi_{S S}\right)$, which is never positive in our model. In the NR scenario, if there is no cost associated with the $R$ allele it experiences no selection pressure, otherwise it would decline over time due to the effect of fitness costs.

In practice, rather than determining the $R$ allele frequency by calculating $\Delta p$ from Eq. (3), we use a simulation approach that involves calculating the frequencies of every genotype and applying the genotype-specific relative fitness values (Eq. 2). This approach allows us to analyze the model in the same way for no release (NR) and the more complicated cases of release of engineered insects (BL or FL); the latter cases involve a complex system of up to 18 difference equations that cannot be readily reduced to expressions in terms of the allele frequencies. 
Release of engineered insects. To model the effects of the release of RIDL males carrying either a bisex-lethal (BL) or female-lethal (FL) construct, we assume that at each generation, as adults emerge prior to mating, mass-reared adult RIDL males are released at a fixed ratio $d$ (the "RIDL release ratio") to the number of males in the wild population (of any genotype) that survived to reproductive maturity. All engineered released males are assumed to be homozygous susceptible to the RIDL construct (SS), and homozygous for the RIDL construct $(L L)$. Wild and released adults mix homogeneously and females mate with wild or released males at random.

Inherent in our model is a proportional release policy, where the number of insects released is proportional to the number of adult males in the current population. An obvious alternative would be a constant release policy, where the same fixed number of RIDL males is released at every generation (which could be described by the "release ratio" of that number to the number of adult males at the start of the program). Implementing the proportional policy would require more sophisticated monitoring to provide data for reasonably accurate prediction of the adult male population at each generation so that the numbers for release can be estimated. We model the proportional policy because working in terms of proportions permits us to study the evolution of allele frequencies independently of population size (so those results are independent of any assumptions about population dynamics). 


\subsection{Population dynamics}

Density-independent populations. $R_{0}$ is the average number of progeny produced per adult pest insect that survive to adulthood in a density-independent population, commonly expressed as female offspring per adult female. For agricultural pests under field conditions, after taking into account factors such as egg hatch rates, predation and pathogens that are common to all genotypes throughout the habitat, $R_{0}$ might typically range from 3 to 11 but could be much higher in some populations (Dyck et al., 2005; Dye, 1984). For NR, BL and FL strategies the (dimensionless) relative population size is determined by

$$
\begin{aligned}
& N_{t+1}=2 R_{0} F_{t} \sigma_{t} \\
& F_{t+1}=R_{0} F_{t} \sigma_{t}^{(F)}
\end{aligned}
$$

where $N_{t}$ is the population size of mature adults at generation $t$ relative to the initial pest density ( $\operatorname{set} N_{0}=1$ ), of which $F_{t}$ are female. The population growth rate is $2 R_{0}$ (each adult female contributes $R_{0}$ male and $R_{0}$ female larvae to the next generation) and $\sigma_{t}$ and $\sigma_{t}^{(F)}$ are the proportions of all offspring or female offspring, respectively, that survive to maturity (simulated by applying the fitness costs of $R$ alleles and the RIDL construct, and the lethal effect of the RIDL construct tempered by the resistance trait, as appropriate to each genotype).

Populations subject to density-dependent processes. Density-dependent processes play an important role in the dynamical behavior of biological populations, and a number of difference equation models to portray them have been described (Bellows, 1981). We adapt a general model of the form 


$$
\widetilde{N}_{t+1}=R_{0} \widetilde{N}_{t} e^{-\alpha \widetilde{N}_{t}}
$$

where $\tilde{N}$ denotes absolute (rather than relative) population size, and $\alpha$ is a measure of the strength of density dependence. Here $1 / \alpha$ is related to the carrying capacity of the habitat, such that if $\alpha=0$ we recover the density-independent model, and in the limit as $\alpha \rightarrow \infty$, $\widetilde{N}_{t+1} \rightarrow 0$ (the carrying capacity approaches zero and nothing survives). This system has equilibria at $\widetilde{N}^{*}=0$ and

$$
\widetilde{N}^{*}=\frac{\log \left(R_{0}\right)}{\alpha}
$$

To make this model dimensionless, we assume that the population starts at the equilibrium in Eq. (7) $\left(\widetilde{N}_{0}=\widetilde{N}^{*}\right)$ and measure the population size relative to that value $\left(N_{t}=\frac{\widetilde{N}_{t}}{\widetilde{N}^{*}}\right)$, so that $N_{0}=N^{*}=1$. It is important for our purpose to distinguish between males and females, so we express population growth in terms of progeny per female as we did in the density-independent model. Finally, we multiply by the survival proportions $(\sigma)$, which implies that the genetically-induced lethality occurs after the density-dependent processes (representing a late-acting rather than early-acting genetic construct).

With parameters and symbols given in Table 1, the relative population size in our densitydependent model is determined by

$$
\begin{aligned}
& N_{t+1}=2 R_{0} F_{t} e^{-\left(\log \left(R_{0}\right) N_{t}\right)} \sigma_{t} \\
& F_{t+1}=R_{0} F_{t} e^{-\left(\log \left(R_{0}\right) N_{t}\right)} \sigma_{t}^{(F)}
\end{aligned}
$$


To ensure that the conclusions we draw with this simple form of density dependence are valid, we have repeated our analyses using a two-parameter density-dependent function (see supplementary material).

\subsection{Simulations}

We assume a naïve population $\left(l_{0}=0\right)$ with genotypes initially in Hardy-Weinberg equilibrium (frequencies $S S w w: q_{0}^{2}, S R w w: 2 p_{0} q_{0}, R R w w: p_{0}^{2}$ ). In the case of RIDL releases, other genotypes ( $L w$ and $L L$ types) arise from matings in the field.

For simplicity, we assume the RIDL construct is fully dominant and completely lethal $\left(\varepsilon_{\text {target }}=1\right)$ and the FL version is lethal to females but not to males; in these simulations,

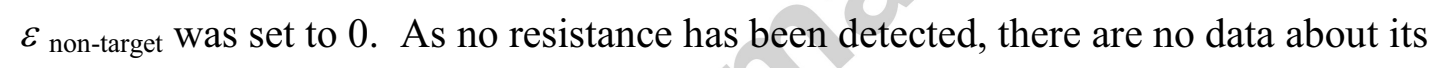
potential properties, so we consider values across the permissible range (the constraints imposed in our model are summarized in Table 1).

We previously showed that lower release ratios than typically employed for the purpose of population suppression could be useful in some cases where the main objective is to manage resistance to an independent control measure such as Bt crops (Alphey et al., 2009; Alphey et al., 2007b). However, in the context of resistance to a RIDL construct, a higher release ratio of RIDL insects would provide both more $S$ alleles to help dilute resistance and greater selection pressure in favor of resistance, so it is not clear what the effect of a higher or lower RIDL release ratio might be. 
We explore the effects of release of RIDL males by simulating different combinations of $\gamma$ and $\psi$ representing varying strength and fitness costs of resistance, over a range of release ratios from 1:10 (one RIDL male per ten males in the wild, 0.1 ) to 1000:1, or a subset thereof. Generally we model a release ratio of 10:1, which could be a realistic ratio for a control program; overflooding ratios quoted in practice (typically 10:1 to 40:1 (Dyck et al., 2005)) may be higher to allow for poor dispersal and other losses during distribution, whereas our release ratio is net of any such effects and all the released insects are assumed to be available for mating. We simulate the frequencies of all genotypes over at least 30 generations to explore transient dynamics and more as necessary (30 up to several thousands) to estimate the value of an equilibrium allele frequency. Those higher numbers are far beyond the realistic practical duration of any release program, but we use them for accurate estimation of equilibria by generating results past any transient behavior to a time when the allele frequencies should have converged.

We generally model initial $R$ allele frequency $p_{0}$ of 0.01 to investigate resistance that is present at perceptible levels in the wild population, or 0.001 to represent a rarer allele that would probably not be readily detectable. We also use an intermediate frequency 0.1 that should be easy to detect in a field population and would cause practical concern, and some much higher values (for example, 0.7 or 0.99 ) to explore the potential reversal of very common resistance. A recent mutation might only have frequency of the order of $10^{-6}$; we do not present results for such very rare alleles as we expect that any trend indicated by results for $p_{0}$ of 0.01 or 0.001 (and other values where simulated) would extend to lower 
values, and because we anticipate that it would take relatively many generations for the impact of such a very rare resistant allele to manifest.

\section{Results}

\subsection{Managing resistance to a fully-lethal genetic construct}

We begin by considering the baseline case where there is no resistance and then investigate the simple cases where resistance is either dominant or recessive, is complete (entirely removes susceptibility to the RIDL construct) and has no fitness costs. Later we progressively relax these assumptions to consider the full range of resistance properties and fitness costs.

Resistance is absent or lethal. There are three situations in terms of model parameters that are equivalent in that they represent the case where there is no resistance in the population: no $R$ allele ( $p_{0}=0$ ), a candidate " $R$ allele" that does not actually confer any resistance property (susceptibility $\gamma_{S S}=\gamma_{S R}=\gamma_{R R}=1$ ) and so is not a resistant $R$ allele, or an $R$ allele that is dominant fully lethal (relative fitness $\psi_{S R}=\psi_{R R}=0$ ) and so never confers any resistance trait. In this baseline case we consider the implications for $R$ and $L$ allele frequencies.

In these circumstances, a resistant $R$ allele $\left(\gamma_{R R}<1\right)$ tends toward (or remains at) zero frequency, i.e. the only stable equilibrium is extinction:

$$
p^{*}=0
$$


As we have no mutation or immigration in our model, no resistant allele ever arises if it is initially absent. A dominant fully lethal allele will not survive into subsequent generations. A candidate allele that turns out to be non-resistant $\left(\gamma_{R R}=1\right)$ is not of interest. For completeness, note that such an allele has no fitness advantage, and will either remain stable (under NR or BL scenarios if it has no fitness costs) or decline to extinction due to dilution and/or fitness costs (in all other situations).

The evolution of $L$ allele frequency differs between BL and FL strategies. With no resistance and BL releases carrying a fully lethal $L$ construct, all progeny of released males are non-viable, so no $L w$ or $L L$ genotypes survive and the $L$ allele does not spread. (There is no introgression of other alleles either, including any $S$ alleles). Frequency $l$ remains zero in all generations (frequencies are measured in adults emerging in the wild, and so exclude the released males).

$$
\mathrm{BL}: \quad l^{*}=0
$$

Under the FL strategy, in the absence of resistance all $L w$ or $L L$ females die (and so all adult females are $w w$ ), but males survive and so the $L$ allele will introgress into the population. The equilibrium $L$ allele frequency can be shown (see supplementary material Appendix A) to be

$$
\mathrm{FL}: \quad l^{*}=\frac{1 / 2 d}{1+d}
$$

This is a stable equilibrium that increases with increasing release ratio. 
Resistance is present. We now consider the situation where a viable resistance trait is present in the wild population $\left(p_{0}>0, \gamma_{R R}<1\right.$ and $\left.\psi_{S R}>0\right)$. The equilibria set out above for no resistance (Eqs. 10-12) are minimum values for allele equilibria in the presence of resistance; they will still apply unless the $R$ allele's properties are such that the fitness advantages outweigh the fitness penalty of the $L$ allele and the effects of dilution by $S$ alleles (which depend on the release ratio $d$ ). This can occur if the $R$ allele is not lethal (fitness $\psi_{R R}$ and perhaps $\psi_{S R}$ not zero) and is effective against the RIDL construct (susceptibility $\gamma_{R R}$ and perhaps $\gamma_{S R}$ below 1). If so, the $R$ allele can persist $\left(p^{*}>0\right)$, and so some target $L L$ or $L w$ genotypes can survive (both sexes are targeted by BL and females are the target for FL), which allows the $L$ allele to persist at higher frequency $\left(l^{*}>0(\mathrm{NR}\right.$, $\mathrm{BL})$ or $\left.l^{*}>\frac{1 / 2 d}{1+d}(\mathrm{FL})\right)$. In a region of parameter space near $\psi_{S R}=\psi_{R R}=0$ and $\gamma_{S S}=\gamma_{S R}=\gamma_{R R}=1$, the $R$ allele's advantages will not be sufficient to achieve this and the $R$ and $L$ allele frequencies will settle at those minimum equilibrium values (Eqs. 10-12). For convenience, we will refer to these minimum equilibrium values (Eqs. 10-12) as "floor" values and to the combinations of susceptibility parameter values where they are the outcome as "floor regions".

With RIDL releases (BL or FL), a proportion $d /(1+d)$ of progeny embryos (prior to the effects of $R$ and $L$ ) will be sired by released SSLL males, and so will have genotype of the form $S_{-} L_{-}$(meaning at least one $\mathrm{S}$ allele, i.e. the $S / R$ type is $S S$ or $S R$, and at least one $L$ allele, i.e. the $L / w$ type is $L w$ or $L L)$. As release ratios would usually be 1:1 or higher ( $d \geq 1$, with realistic $d>>1$ for a suppression or elimination program), the majority of 
embryos will have genotype of that form. We therefore expect that the fitness costs for $S R$ heterozygotes (fitness $\psi_{S R}$ ) and the effectiveness of heterozygote resistance (susceptibility $\gamma_{S R}$ ) will have more influence on the evolution of $R$ allele frequency, and hence on $L$ allele frequency, than those of $R R$ homozygotes do. This effect is more pronounced as the release ratio, $d$, increases.

Below, we confirm and expand on these general deductions by progressively relaxing assumptions about the genetic properties of resistance.

Complete dominant no-cost resistance. The strongest combination of resistance properties is an $R$ allele with no fitness costs (fitness $\psi_{S R}=\psi_{R R}=1$ ), conferring resistance that is complete (i.e. negates any effects of the $L$ allele) and dominant (susceptibility $\gamma_{S R}=\gamma_{R R}=0$ ). Such an allele has the greatest potential to spread among the population in the presence of the RIDL construct, and simulations show that it spreads rapidly when RIDL releases are in progress (Fig. 1a,b, BL and FL). The $R$ allele equilibrium values are above $1 / 2$ for $\mathrm{BL}$ and above $1 / 3$ for FL releases, which is abundant enough to allow the $L$ allele to spread very quickly to fixation (Fig. 1c,d). FL is better than BL at controlling resistance (Fig. 1a,b) and the $L$ allele spreads marginally faster with FL than BL (Fig. 1c,d), because the female-specific $L$ allele is inherited through all males not just resistant males. In the absence of the RIDL construct, the $R$ allele encounters no selection pressure and will remain constant (Fig. 1a,b, NR). 
Similar patterns are observed across a range of values for the initial $R$ allele frequency.

Starting with $p_{0}$ at 0.1 (Fig. 1a,c), 0.01 or 0.001 (Fig. 1b,d), all yield the same broad pattern and identical equilibrium frequencies. Even from near fixation $\left(p_{0}=0.99\right)$, the same equilibria result, with the $R$ allele declining rapidly to that value and the $L$ allele spreading very rapidly from 0 to fixation.

The shapes of these allele frequency curves against time are examples of the patterns that can be seen more generally, not only for complete, dominant, no-cost resistance. The first two generations of RIDL releases (BL or FL) into a naïve population may exhibit distinctive changes (e.g. clearly visible in Fig. 1d FL), because those are when $L w$ (first generation) and $L L$ (second generation) individuals first appear among the population emerging in the wild. Apart from this, the frequency of a spreading $R$ allele is consistent with the sigmoid pattern often associated with resistance generally: a potentially long period (for a very rare allele) with little change, followed by a period of rapid increase, then leveling off approaching the equilibrium frequency (Fig. 1a,b, BL, FL). The $R$ allele never reaches fixation with RIDL releases because of the dilution effect of adding new $S$ alleles at each generation, but the equilibrium frequency can potentially be close to 1 (see later examples). The same general patterns can be observed in the frequency of a RIDL genetic construct ( $L$ allele), except that the $L$ allele can reach fixation with sufficiently fit and effective resistance (Fig. 1b,d), and if the $R$ allele starts at high frequency the $L$ allele can at first increase to a higher level and then decline to its equilibrium value. 
The values of $R$ allele equilibria under BL and FL strategies depend on the release ratio. The dominant $R$ allele has a significant advantage in the presence of the $L$ allele and spreads as far as it can subject to dilution (which effect is stronger at higher release ratios).

As release ratio $d$ increases, $p^{*}$ decreases toward $1 / 2$ for BL or $1 / 3$ for $\mathrm{FL}$, and this equilibrium is approached more quickly (Fig. 2a,b). A higher release ratio also increases the rate of spread of the $L$ allele to fixation (Fig. 2c,d). Very similar patterns are seen starting from $p_{0}=0.1$ (Fig. 2 illustrates $p_{0}=0.001$ ), except that the alleles reach their equilibria more quickly, especially with the lower release ratios. The limits for $p^{*}(1 / 2$ with BL, $1 / 3$ with FL) can be deduced from the genetics (see supplementary material Appendix B).

Complete recessive no-cost resistance. If the $R$ allele has no fitness costs (fitness $\left.\psi_{S R}=\psi_{R R}=1\right)$ and gives complete resistance $\left(\gamma_{R R}=0\right)$ but is recessive (susceptibility $\left.\gamma_{S R}=\gamma_{S S}=1\right)$ rather than dominant, this is weaker resistance than the dominant equivalent. The $R$ allele only has an effect in homozygous form.

With BL release, all progeny of released males are $S S L_{-}$or $S R L_{-}$and so are non-viable, and there can be no introgression of $S$ or $L$ alleles into the wild population. The $R$ allele remains at its original frequency because $R R L_{-}$genotypes (i.e. $R R L w$ or $R R L L$ ) never arise to create selection pressure, and the $L$ allele does not enter the population (Fig. 3, BL). This holds true for all recessive resistance, whether or not it is complete $\left(\gamma_{R R} \geq 0\right)$. 
With FL release, all female progeny of released males are $S S L_{-}$or $S R L_{-}$and so are nonviable, but there is introgression of $S$ or $L$ alleles through the male line. With release ratio 10:1, the dilution effect of $S$ alleles from released RIDL males is strong enough that the $R$ allele declines to extinction (Fig. 3a), which means that the $L$ allele settles at its floor equilibrium value (Eq. 12) and does so rapidly at this release ratio (Fig. 3b). Increasing the release ratio increases $l^{*}$, with a limit of 0.5 as $d \rightarrow \infty$.

The equilibria, and the finding that there is no change in $R$ allele frequency with $\mathrm{BL}$, are independent of initial $R$ allele frequency. Fig. 3 illustrates $p_{0}$ of 0.001 . With $p_{0} 0.1$ broadly similar patterns over time are observed. Even with very high $p_{0} 0.99$ the equilibria (or no change) are the same, but the transient dynamics differ. With FL releases, $R$ frequencies decline monotonically from that high level and the $L$ allele frequency increases at first (reaching a maximum exceeding 0.62 in the 15 th generation) before declining to the same equilibrium value.

Incomplete no-cost resistance. Resistance might reduce the fitness penalty of the RIDL construct rather than cancel it, i.e. resistance might be incomplete. The effectiveness of a dominant $R$ allele with no fitness costs (fitness $\psi_{S R}=\psi_{R R}=1$ ) varies with the susceptibility of resistant insects $\left(0 \leq \gamma_{R R}=\gamma_{S R} \leq 1\right)$. The closer that susceptibility to the RIDL construct is to 0 (i.e. the more effective resistance is), the faster both $R$ and $L$ alleles spread (Fig. 4). With FL releases, the $R$ allele equilibrium is lower with less effective resistance (Fig. 4b). Under the BL strategy there is very little variation in the $R$ allele equilibrium (with releases at 10:1 $p^{*}$ remains close to 0.52 , Fig. 4 a). For both strategies, the additional survival of $L$ 
alleles with stronger resistance can cause a significant increase in their frequency (Fig. $4 \mathrm{c}, \mathrm{d})$.

When we allow susceptibility to the RIDL construct to range over all permitted values $\left(0 \leq \gamma_{R R} \leq 0 \gamma_{S R} \leq 1\right)$ with no assumptions about dominance, we see that this variation generally has more effect on $l^{*}$ than on $p^{*}$ (Fig. 5). As predicted, varying $S R$ susceptibility has a greater effect on equilibria than varying $R R$ susceptibility; the slopes of the $p^{*}$ and $l^{*}$ surfaces are generally steeper for fixed $\gamma_{R R}$ than for fixed $\gamma_{S R}$ (this is more visible for $l^{*}$ ). Except for the special case of recessive resistance $\left(\gamma_{S R}=1\right)$ with BL (where $p^{*}=p_{0}$, which is 0.01 in Fig. 5a), at realistic release ratios equilibria are independent of initial $R$ allele frequency (we modeled $p_{0}$ of 0.01 (Fig. 5), $0.1,0.7$ and 0.99 with $d=10$ ), and where resistance is recessive $\left(\gamma_{S R}=1\right)$ the $R$ and $L$ alleles tend to their floor values (Eqs. 10-12) (Fig. 5b-d). Where resistance is complete and dominant (i.e., one or more copies of the $R$ allele prevent any susceptibility to the genetic lethality $\gamma_{S R}=\gamma_{R R}=0$ ), the $R$ allele spreads as far as it can subject to the effects of dilution (Fig. 5a,b) and the $L$ allele goes to fixation (Fig. 5c,d). The $L$ allele also goes to fixation for parameter values in a region around that corner of parameter space ( $\gamma_{S R}$ and $\gamma_{R R}$ near zero), where the lethality of the RIDL construct is still overcome by the very effective resistance compared with the effects of dilution. What happens in between is slightly different for BL and FL strategies.

In the BL scenario, for any given $\gamma_{R R}$, as heterozygote susceptibility $\gamma_{S R}$ increases toward higher values (weaker heterozygote resistance), $p^{*}$ increases (Fig. 5a) and $l^{*}$ decreases 
(Fig. 5c) toward extinction (its floor, Eq. 11). This is the result of fewer $S R L_{-}$individuals surviving; this reduces the effect of dilution by $S$ alleles and introgression of $L$ alleles from released RIDL males because that is the genotype of their potentially resistant offspring. For given $\gamma_{S R}$, as homozygote susceptibility $\gamma_{R R}$ increases, $p^{*}$ (Fig. 5a) and $l^{*}$ (Fig. 5c) both decrease (although not by much) because reduced survival of $R R L_{-}$types reduces the frequency of $R$ alleles a little. With lower release ratio $(d=5)$, the equilibrium surfaces are similar, but with higher $p^{*}$ values due to less dilution, and lower $l^{*}$ values, with $L$ allele reaching fixation in a slightly smaller region of parameter space near the corner $\gamma_{S R}=\gamma_{R R}=0$ than it did with $d=10$. At even lower release ratio $(d=1)$ those results remain robust, although the $p^{*}$ surface appears a little less flat near one edge $\left(\gamma_{R R}=0\right)$, and again, the equilibrium results are the same with $p_{0} 0.01$ as with 0.99 .

In the FL case, the outcomes are more sensitive to some parameter values. As in the BL case, for any given $\gamma_{S R}$, as homozygote susceptibility $\gamma_{R R}$ increases, the equilibria $p^{*}$ and $l^{*}$ decrease a little (Fig. 5b,d), and over most of the range of values, holding $\gamma_{R R}$ fixed and increasing $\gamma_{S R}$ (less effective heterozygote resistance) reduces $l^{*}$ towards its floor (Eq. 12) (Fig. 5d). This is consistent with fewer target $S R L_{-}$females surviving and so reducing the introgression of $L$ alleles from released RIDL males through their female progeny. There is distinct interesting behavior in one corner region, where $\gamma_{R R}=0$ (homozygotes have complete resistance and so are as fit as $S S$ genotypes), and $\gamma_{S R}$ is near 1 (resistance is closer to recessive than dominant); this situation where $\gamma_{R R}=0$ is investigated fully in the supplementary material. With release ratio $10: 1$, there is little change in the $R$ allele 
equilibrium frequency $p^{*}$ over most of the surface (Fig. 5b). Holding $\gamma_{R R}$ constant, increasing $\gamma_{S R}$ can slightly increase or decrease $p^{*}$ at different values of $\gamma_{S R}$, and again there is atypical behavior in the same region. With a release ratio 10:1, the $p^{*}$ and $l^{*}$ equilibria surfaces are the same regardless of initial $R$ allele frequency (identical equilibrium results for $p_{0} 0.01$ and 0.99$)$. With a lower release ratio $(d=5$ or 1 , supplementary Fig. S1), the surfaces appear similar to Fig. 5b\&d except that the $l^{*}$ floor is lower (Eq. 12) and the $p^{*}$ and $l^{*}$ floor regions extend further from the edge where $\gamma_{S R}=1$; the lower release ratio, the greater the observed effects. The outcome in the atypical corner is affected by initial $R$ allele frequency; when $p_{0}$ is higher there are more pronounced peaks in the region of interest (see supplementary material results).

The impact of fitness costs of resistance. Where the $R$ allele has fitness costs, recessive resistance $\left(\gamma_{S R}=1\right)$ is no longer a special case for the BL strategy; the costs cause the $R$ allele to decline to extinction rather than remain unchanged at its initial frequency. The existence of fitness costs for resistance to a FL RIDL construct removes the interesting behavior discussed above from the region of parameter space where $\gamma_{S R}$ is near 1 (resistance is nearly recessive) and $\gamma_{R R}=0$ (homozygote resistance is complete). (We modeled $p_{0}$ of $0.01,0.1$ or 0.8 with $d=10$, and $p_{0}$ of 0.01 with $d=1$, to confirm both these findings). As a result, the BL and FL equilibrium surfaces against susceptibility to RIDL are similar in shape (but not scale) and behavior, so we illustrate only FL. 
With both BL and FL strategies, as fitness costs associated with the $R$ allele increase, especially those for $S R$ heterozygotes, the $R$ allele goes extinct for a greater range of heterozygote susceptibility values, and where it persists it tends toward a lower equilibrium (compare Fig. 5b,d no costs, Fig. 6a,c low costs and Fig. 6b,d high costs). This reduction in $p^{*}$ is smaller for more effective $R$ alleles (low values of susceptibility $\gamma_{S R}$ and $\gamma_{R R}$ ).

Resistance with high heterozygote susceptibility $\gamma_{S R}$ is not sufficiently effective, in the presence of fitness costs, for the $R$ and $L$ alleles to persist above their floors (Eqs. 10-12) and those floors extend over a larger region (mostly defined by a $\gamma_{S R}$ threshold).

A resistant allele that is very costly (high fitness penalty in heterozygotes and homozygotes) would be harder to control when it is very abundant than when it is rare. However, such a high-cost allele would not be expected to be abundant in practice, because of the selection pressure. With 10:1 release ratio, $p_{0}$ only makes a difference to $p^{*}$ and $l^{*}$ for the most costly $R$ alleles ( $\psi_{S R}$ and $\psi_{R R}$ near zero); for such alleles, higher $p_{0}$ gives a smaller floor region (the edge occurs at higher $\gamma_{S R}$ ) but does not alter equilibrium values elsewhere. That is, for costly resistance with given susceptibility, $p_{0}$ determines whether the $R$ and $L$ alleles will persist above their floors (Eqs. 10-12) but, if they do, it does not change the equilibrium values at which they will persist. These results suggest some kind of bifurcation of surfaces (floor versus persistent equilibria) for each of the $R$ and $L$ alleles for a given release ratio, with $p_{0}$ determining on which surface the result lies. The critical $p_{0}$ appears to be moderated by the release ratio and fitness cost of resistance, and if it does not fall between 0 and 1, floor values will result either at all values of susceptibility or only 
for recessive resistance (the edge where $\gamma_{S R}=1$ ). At the edge of the floor, the transition can be continuous (Fig. 6a,c) or discontinuous (Fig. 6b,d).

A lower release ratio (1:1 instead of the 10:1 shown in Fig. 6) reduces the $\gamma_{S R}$ threshold thereby enlarging the floor regions (for example, with $d=1$ and costly resistance, floors for both $R$ and $L$ alleles extend across the whole range of susceptibility values for BL and FL). This occurs because there is less selection pressure in favor of the $R$ allele at lower release ratios. With effective resistance (on the low susceptibility $\gamma_{S R}$ side of that threshold) the $L$ allele goes to fixation over a smaller range of susceptibility values and $p^{*}$ is higher. The $R$ allele persists at higher equilibrium value because the lower release ratio lessens the effect of dilution by $S$ alleles from released males.

These effects of reducing the release ratio suggest that for any given set of parameter values $(\gamma, \psi)$ and initial $R$ allele frequency there is a critical release ratio, below which the $R$ allele tends to extinction and the $L$ allele tends to extinction (BL) or its non-zero floor (FL). This is borne out by simulations. This critical release ratio depends on the genotypes' relative fitness values, with regard to both costs and efficacy of resistance and, for some parameter combinations, the initial $R$ allele frequency. For example, with $\gamma_{S R}=0.9, \gamma_{R R}=0.4$, $\psi_{S R}=0.4$ and $\psi_{R R}=0.3$, (nearly recessive incomplete resistance with nearly dominant, moderately high fitness costs), the critical release ratio for FL is roughly $37: 1$ with $p_{0}=0.001$ or 0.9 , but the critical release ratio for $\mathrm{BL}$ is $58: 1$ with $p_{0}=0.001$ or $47: 1$ with $p_{0}=0.9$. More effective resistance and/or lower fitness costs give a lower critical release 
ratio, which could be significantly below the release ratios expected for a suppression or elimination program.

Homozygote costs and susceptibility have little effect compared with heterozygote costs and susceptibility. For example, having moderate or high homozygote fitness $\operatorname{costs} \psi_{R R}=0.4$ or 0.1 , does not change the critical release ratios of roughly $0.37: 1$ for FL and 0.41:1 for BL, with $\gamma_{S R}=0.3, \gamma_{R R}=0.1$ and $\psi_{S R}=0.9$ (partially dominant, very effective resistance with near recessive fitness costs) and $p_{0}=0.001$ or 0.9 . Relative fitness in homozygotes is unimportant and it is mainly the phenotype in heterozygotes that determines whether resistance spreads.

Regardless of $p_{0}$, some combinations of parameter values can give rise to a large discontinuity at the critical release ratio while others do not. For example, starting BL releases when $p_{0}=0.9$, with $\gamma_{S R}=0.9, \gamma_{R R}=0.4, \psi_{S R}=0.9$ and $\psi_{R R}=0.1$, the critical release ratio (below which $p^{*}=l^{*}=0$ ) is roughly $4.2: 1$, and the slightly higher release ratio 4.5:1 results in very low equilibria $p^{*}=0.0087$ and $l^{*}=0.0017$. When alleles are this rare (and they become so fairly quickly $-p$ reduces from 0.9 to just above 0.05 in 30 generations) a release ratio a little over the critical value need not be cause for practical concern. However, with $\gamma_{S R}=0.3, \gamma_{R R}=0.1, \psi_{S R}=0.4$ and $\psi_{R R}=0.3$, the critical release ratio is $2.1: 1$, but releases at 2.15:1 result in very much higher equilibria $p^{*}=0.6009$ and $l^{*}=1$. Even so, as results in the next section show, the spread of a 
resistant allele will not necessarily have a significant impact on suppression of the population.

\subsection{Population control and resistance management}

For practical purposes, it is not the frequency of a resistant allele that is directly of interest, but the size of the pest population. We explored the interaction between the evolution of allele frequencies and the impact on the relative size of the pest or vector population. We also examined the relationship with the insect's net reproductive rate $R_{0}$ (the per capita rate of population growth in a generation), employing a higher release ratio for a pest with higher growth potential. We limited these investigations to situations where the $R$ allele will not decline to extinction, because that is where concern would focus, and compared the outcomes with the equivalent release regimes in the absence of any resistance. We selected release ratios that would lead to a successful program (local elimination of the pest) in the absence of resistance.

With no release and bisex-lethal RIDL release strategies, the females are always half of the population. Under the female-lethal RIDL release strategy this is not true; the relative number of females is less than half the relative total population size because females are targeted and are killed off disproportionately. The effect of resistance is qualitatively similar whether measuring the total number of insects or the number of females (illustrated in supplementary Fig. S3, compare panels a \& d).

Under our simulated proportional release policy, female-lethal releases appear to perform better in terms of relative population size than bisex-lethal releases at the same release ratio 
(Figs. 7, S3 \& S4). This occurs as the number of insects released is proportional to the number of males emerging in the wild; the BL strategy kills males and females in equal numbers but FL strategy kills only females. The same release ratio for both strategies involves more insects being released under the FL strategy than the BL strategy, so the two are not directly comparable.

Density-independent populations. The presence of a resistant allele that spreads to nonzero equilibrium has greater effect on the population size if it is a stronger $R$ allele (with low fitness costs and low susceptibility to the lethal genetic construct) and/or if the insect has a high per capita population growth rate. A weaker $R$ allele (with a smaller reduction in susceptibility and higher fitness costs) can have a fairly small impact on population control, by marginally slowing the rate at which releases drive the pest to local elimination (supplementary Fig. S3a). If the pest has a low population growth rate, such resistance can have negligible impact on population management (with lower $R_{0}$ and $d$ values, as used in Fig. S3b, the results with and without resistance are indistinguishable on the equivalent of Fig. S3a until the relative population size is below $10^{-20}$ ). A stronger $R$ allele has a greater effect on population size, although with lower $R_{0}$ the population may remain suppressed below its original size for many generations (Fig. S3b). At worst, at a release ratio that would be very effective in the absence of resistance, strong resistance in a pest with higher $R_{0}$ can mean that instead the releases only slow the rate of explosion of the population (Fig. $\mathrm{S} 3 \mathrm{c})$. In all instances we modeled, releases did at least as well as no release: even complete dominant no-cost resistance (fitness $\psi_{S R}=\psi_{R R}=1$, susceptibility $\gamma_{S R}=\gamma_{R R}=0$ ) resulted in significantly slower population growth than for the uncontrolled population. 
As described above, the initial $R$ allele frequency does not make a difference to the equilibrium $R$ or $L$ allele frequencies for most combinations of fitness costs and susceptibility. If the allele starts at a much higher frequency its impact on the population size in the early generations could potentially affect the relative population size after the equilibria have been reached. However, we generally observed only a slight slowing of population decline or increase of population explosion (by a small number of generations). This might or might not be large enough to have a significant economic or public health effect. For example, with parameters as in Fig. S3, the largest difference was with the stronger resistance with the higher growth rate (Fig. S3c), where the population became more than a million times its original size after 14 generations from $p_{0}=0.9$, compared with 19 from $p_{0}=0.01$. The impact was much smaller for the other panels.

Populations subject to density-dependent processes. Broadly, we draw the same general conclusions about the impact of resistance to the RIDL construct when there are densitydependent processes operating as for density-independent populations. The main difference is that the population is assumed to start at equilibrium, so the relative population size will remain at 1 with no releases (Fig. 7). The RIDL construct fitness penalty takes effect after density-dependent mortality, because of its late-acting lethality, and successful RIDL releases can drive the population to local elimination. Where resistance disables the efficacy of the RIDL construct, the population will return to equilibrium densities. 
Density-dependent processes do not alter the prediction that a spreading resistant allele has greater effect on the population size if it is a stronger (low susceptibility and low cost) $R$ allele and/or if the insect has a high per capita population growth rate. The presence of a weak $R$ allele can mean releases take slightly longer to drive the pest to local elimination (Fig. 7a); with low population growth rate the impact on population control might be negligible (e.g. as Fig. 7a except with the lower $R_{0}$ and $d$ values). A strong $R$ allele is more detrimental for the effectiveness of releases, although with lower $R_{0}$ the initial suppression, while resistance is still uncommon, is enough to keep the population below its original size for many generations (Fig. 7b). At high release ratios, strong resistance spreading in a pest with higher $R_{0}$ might only suppress the pest for a few generations before the population recovers to near natural equilibrium densities (Fig. 7c). If that $R$ allele is initially widespread (e.g. as Fig. 7c but with $p_{0}=0.9$ instead of 0.01 ) that initial suppression phase does not occur and pest densities move quickly to that new level just below the natural equilibrium.

We obtained qualitatively similar results with a two-parameter density-dependence process (see results in supplementary material and Fig. S4). A weaker $R$ allele (with high susceptibility to the genetic lethality and some fitness costs) can make negligible difference to the effectiveness of controlling a low growth rate population, and the impact of resistance increases with the strength of the $R$ allele and the population growth rate.

In all instances modeled, we found that releases reduced the population below the natural equilibrium. Even complete dominant no-cost resistance (fitness $\psi_{S R}=\psi_{R R}=1$, 
susceptibility $\gamma_{S R}=\gamma_{R R}=0$ ) resulted in population densities slightly below equilibrium and could exhibit suppression temporarily while resistance was uncommon. (For example, with other parameters as in Fig. $7 \mathrm{~b}$ i.e. $p_{0} 0.01, d 10$ and $2 R_{0} 6$, population size declined to 0.03 relative to equilibrium in $3(\mathrm{BL})$ or $4(\mathrm{FL})$ generations, then recovered to $0.45(\mathrm{BL})$ or 0.41 (FL) by the 17th generation.) The temporary period of effective suppression can last longer if starting with a rarer allele.

\section{Discussion}

We have shown that a RIDL approach could potentially limit the effects of biochemically based resistance against the RIDL genetic construct ( $L$ allele) engineered into the released strains, due to a built-in dilution mechanism. Resistant alleles are unable to spread to fixation, although they can become more common than the alternative susceptible allele. However, the evolution of resistant allele frequency is subject to a complicated mix of competing forces.

A resistant allele with no fitness costs has a net advantage in a population containing the RIDL genetic construct, the magnitude of which depends on the effectiveness and dominance of resistance (which we express in terms of genotype susceptibility to the RIDL construct). That advantage depends on the frequency of the RIDL allele, which in turn depends on the release ratio. However, whilst a higher release ratio increases the frequency of the $L$ allele and thereby acts in favor of resistance, it also increases the dilution of the gene pool by susceptible alleles to reduce resistance. The existence of fitness costs associated with resistance adds another complication by affecting the direction and 
magnitude of the resistant allele's net advantage or disadvantage. This is a multidimensional problem for which several parameter values cannot reasonably be predicted because resistant alleles are likely to be rare or non-existent at the commencement of a release program, and may be difficult to measure or estimate accurately even if resistance has been identified, especially if resistance is recessive or nearly recessive so that it is only observed in homozygous form.

In the absence of resistance, increasing the ratio of released RIDL males to males in the wild has a beneficial impact on the size of the pest population. As with classical SIT, there is a critical release ratio above which the size of the population will be reduced (Dyck et al., 2005; Phuc et al., 2007; Thomas et al., 2000). Here we have identified that there is another critical release ratio below which resistance will be prevented from spreading through the population and the RIDL construct will not spread among individuals of the target sex(es). Clearly, there may be situations where those two requirements are incompatible, which arise when resistance is more effective and/or has lower fitness costs. However, the population might still be effectively controlled even if resistance becomes common, and with weak resistance that is initially rare there may even be little practical impact for population control. Stronger, fitter resistance has greater potential to be detrimental for the aim of reducing population size.

Stronger resistance (with low fitness costs and low susceptibility to the lethal genetic construct) is the main threat to the success of a genetic control release program. Such resistant alleles have greater selection pressure in their favor and tend to spread very 
rapidly, even from low levels. At worst, against target insects that have little or no regulation by density-dependent processes, the presence of such resistance should therefore become apparent quickly, as monitoring data would reveal a clear detrimental change in the rate of population growth after a promising start. Similarly, population monitoring should identify such resistance among populations that are subject to density-dependent mortality, as a new equilibrium density would be reached fairly rapidly. In practice, though, preimplementation preparatory trials should be expected to detect such fit and effective resistant alleles, unless they are very rare. All RIDL strains incorporate a heritable genetic marker that allows easy identification, so survival of RIDL heterozygotes ( $L w$ individuals) should be readily detected from trapping data during release trials. Laboratory trials involving crosses with the wild population might potentially identify resistant alleles, present in the field, at a much earlier stage. The detection threshold could be of the order of 0.01 allele frequency or lower, depending how recessive the resistance trait is (a resistance effect in $S R$ heterozygotes is easier to detect).

Some of the low release ratios that we have modeled would not intentionally be used in an actual population control program. However, these values might be relevant outside the program's target area if there are regions to which engineered insects could emigrate. It is known that, with density-dependent populations, SIT releases can in theory have unintentional consequences - sometimes detrimental - in neighboring areas, although a related modeling study suggests this would not occur with (bisex-lethal) late-acting RIDL strategies (Yakob et al., 2008). Our findings here suggest that any resistance to the genetic construct in areas of low actual release ratio would be less likely to spread, because of the 
lower favorable selection pressure; it might therefore be expected to interfere less with population reduction outside the target region, than inside it. This is unlike resistance to chemical insecticides, where lower doses (such as spray drift, or early prototype "mediumdose" GM crops) tend to encourage resistance by allowing survival of partially resistant individuals. A model incorporating spatial aspects would be required to investigate this matter fully.

We have not simulated the situation where the RIDL genetic construct is not fully lethal $\left(\varepsilon_{\text {target }}<1\right)$. We might reasonably expect a trade-off between population suppression and resistance management, but the competing selective forces acting on the evolution of resistance might lead to counterintuitive results, so this could be worthy of exploration. We did not simulate scenarios where the female-lethal RIDL construct has a modest fitness cost in males $\left(\varepsilon_{\text {non-target }}>0\right)$. This also could be potentially interesting in conjunction with the population dynamics.

We assumed that the insects released are homozygous susceptible. The dynamics could be different if quality control processes are not able to ensure this. In particular, if resistance is of low dominance, it might be that the release population includes a small number of heterozygous susceptible RIDL males ( $S R L L$ types as well as $S S L L$ ). In principle our model could be adapted to explore this issue.

Compared with the proportional release policy of our model, a constant release policy does not enable population genetics to be studied separately from the population dynamics, so 
some population growth assumptions have to be made, however simple. If the release ratio of released males to initial population of males, say, is high enough to reduce the population initially, in subsequent generations the ratio of released males to actual males in the wild increases. This could mean that the resistance becomes more able to spread as the effective release ratio increases, which in turn could impact the population size. This adds a further layer of competing influences above those encountered in our proportional policy model. In practice, what is implemented might be somewhere between those two, with a larger release quantity at first, reduced in one or more steps as the population declines.

Some mosquito populations vary with environmental factors such as rainfall. Seasonal variation could be modeled by varying the carrying capacity over time. With our proportional release policy, the population genetics are independent of the population dynamics, so our conclusions about allele frequency evolution would not change (unless the resistant phenotype was environmentally dependent, for example, with reduced ability of eggs to survive desiccation). With a constant release policy, the effective release ratio would vary (being at its minimum when the population is at its seasonal peak, and conversely), and seasonality would vary the balance of competing selective forces.

Releasing fertile, susceptible, wild type insects would allow introgression of susceptible alleles into the population in a similar manner to release of RIDL insects. However, given the difficulty of separating wild males from females on an industrial scale, both sexes would probably have to be released and any released fertile females would contribute to population growth. Susceptible alleles would be inherited through both sexes and none of 
the progeny would suffer reduced survival (unlike progeny of RIDL males), so this should be more effective at reducing the frequency of resistant alleles than equivalent RIDL releases. We could incorporate wild type releases into our models, to explore combinations of release strategies to manage resistance and maintain the efficacy of an established RIDL release program. In theory, we could also consider the sequential or alternating use of bisex-lethal RIDL releases and female-lethal RIDL releases, with one being used to combat unrelated resistance to the other while still managing the population size. This would only be appropriate if the two genetic constructs involved had independent modes of action and there was no cross-resistance. Given the molecular basis of the RIDL strains currently in existence or under development (Fu et al., 2007; Fu et al., 2010; Gong et al., 2005; Phuc et al., 2007; Stainton et al., unpublished data; Thomas et al., 2000), these may or may not be reasonable assumptions depending on the particular combination of BL and FL strains used.

Our model could be modified to investigate further questions relevant to resistance management strategies. Any resistant allele would likely be very rare in a natural population, and stochastic effects would be expected to have implications at low frequencies. For example, with a stochastic model we could explore the probability that a resistant allele will go extinct within a specified number of generations. "Behavioral resistance" (see Introduction), rather than biochemical resistance, would require us to replace our assumption of random mating, which would potentially result in different conclusions. We note that a broadly similar model has also been used to simulate the intentional spread through a population of an allele resistant to a lethal gene, as a means of 
driving an anti-pathogen gene through a vector population, with a "killer" gene conferring an advantage to a "rescue" gene to which would be linked a genetic construct that inhibits transmission of a target pathogen (Gould et al., 2008).

A potential concern about releasing GM insects into the wild is that the inserted DNA may have unforeseen consequences. This has particularly been raised regarding strategies that aim to replace a wild population, for example with a version engineered to be unable to transmit a pathogen. One advantage claimed for genetic SIT strategies over population replacement strategies is that autocidal engineered insects are programmed to die and therefore the lethal genetic construct should die out if releases cease. This relies on the construct having some fitness costs even if it does not retain its efficacy. We have shown that resistance to the construct might significantly increase its frequency. In theory, there are combinations of genetic properties of resistance for which the construct could eventually be driven to fixation; that scenario is highly unlikely to be played out in real life because the release program could be stopped if substantial resistance were detected, long before the RIDL construct became common, and the wild type would reinvade through immigration.

It is remarkable that a fairly simple two-locus genetic system with forcing (from releases) can produce such a complicated array of potential behaviors. Some combinations of genetic properties of resistance can generate unexpected results, especially with the femalelethal strategy, and small changes in a single parameter can make the difference between resistance going extinct and resistance spreading so widely as eventually to render the 
lethal construct largely ineffective. However, the theoretical equilibrium results are not always relevant to planning suppression or elimination programs, where it is short to medium term behavior (the transient dynamics) that is important. Ultimately, it is the effect of the resistance on the population size that matters and that can vary from negligible to substantial impact on the results of a release program, with strong (effective and low-cost) resistance and high growth-rate insects being harder to control. Fortunately, those stronger resistance alleles are the ones that are easier to detect in the population either during preparatory trials or during early stages of a release program.

Under an envisioned RIDL release program, it would be important to detect any resistance in the pest population that was capable of having a significant detrimental impact on the program's effectiveness in time to take remedial action. Our population dynamic models predict a clear change in the pattern of pest densities (for example, Figs. 7c \& S3c) as such resistance begins to spread. A properly designed monitoring strategy would be alert to such changes. Wild-caught individuals could then be used to screen for cross-resistance against a panel of available RIDL strains that use different lethal mechanisms, so that operations (or further trials, if this occurred before full implementation) could switch to deploying an appropriate alternative RIDL line before significant lasting loss of efficacy could result. 


\section{Contributors}

All authors designed the study and analyzed the results. NA performed the detailed analysis and simulations and wrote the manuscript. MBB and LA edited the manuscript. All authors have approved the final article.

\section{Disclosure statement}

LA is an employee and NA was a collaborative DPhil/PhD student of Oxitec Limited, which therefore provided salary and other support for the research program. Those authors have shares or share options in Oxitec Ltd (LA) or an indirect interest therein (NA). Both Oxitec Ltd and the University of Oxford have one or more patents or patent applications related to the broader subject of this paper.

\section{Acknowledgements}

This work was supported by the Royal Society (through a Royal Society University Research Fellowship to MBB), the UK Biotechnology and Biological Sciences Research Council (BB/C512702/1, MBB; BBS/S/J/2005/12055, NA) and by a grant to the Regents of the University of California from the Foundation for the National Institutes of Health through the Grand Challenges in Global Health initiative (LA).

\section{Role of the funding sources}

None of the funding sources had any role in study design, the collection, analysis or interpretation of data or results, the writing of the manuscript or the decision to submit it for publication. 


\section{References}

Alphey, L., Nimmo, D., O'Connell, S., Alphey, N., 2007a. Insect population suppression using engineered insects. In: Aksoy, S., (Ed.), Transgenesis and the management of vector-borne disease. Landes Bioscience, Austin, Texas.

Alphey, L. S., 2007. Engineering insects for the Sterile Insect Technique. In: Vreysen, M., et al., Eds.), Area-wide control of insect pests: from research to field implementation. Springer, Dordrecht, The Netherlands, pp. 51-60.

Alphey, N., Bonsall, M. B., Alphey, L., 2009. Combining pest control and resistance management: synergy of engineered insects with Bt crops. J Econ Entomol 102, 717-732.

Alphey, N., Coleman, P. G., Donnelly, C. A., Alphey, L., 2007b. Managing insecticide resistance by mass release of engineered insects. J Econ Entomol 100, 1642-1649.

Atkinson, M. P., Su, Z., Alphey, N., Alphey, L. S., Coleman, P. G., Wein, L. M., 2007. Analyzing the control of mosquito-borne diseases by a dominant lethal genetic system. Proc Nat'l Acad Sci USA 104, 9540-9545, doi:10.1073/pnas.0610685104.

Bellows, T. S., Jr, 1981. The descriptive properties of some models for density dependence. Journal of Animal Ecology 50, 139-156.

Dyck, V. A., Hendrichs, J., Robinson, A. S. Eds.), 2005. Sterile Insect Technique: Principles and practice in Area-Wide Integrated Pest Management. Springer, The Netherlands.

Dye, C., 1984. Models for the population dynamics of the yellow fever mosquito, Aedes aegypti. Journal of Animal Ecology 53, 247-268. 
ffrench-Constant, R. H., 2007. Which came first: insecticides or resistance? Trends in Genetics 23, 1-4, doi:10.1016/j.tig.2006.11.006

Fryxell, K. J., Miller, T. A., 1995. Autocidal biological control: a general strategy for insect control based on genetic transformation with a highly conserved gene. J. Econ. Entomol. 88, 1221-1232.

Fu, G., Condon, K. C., Epton, M. J., Gong, P., Jin, L., Condon, G. C., Morrison, N. I., Dafa'Alla, T. H., Alphey, L., 2007. Female-specific insect lethality engineered using alternative splicing. Nat Biotech 25, 353-357, doi:10.1038/nbt1283.

Fu, G., Lees, R. S., Nimmo, D., Aw, D., Jin, L., Gray, P., Berendonk, T. U., White-Cooper, H., Scaife, S., Phuc, H. K., Marinotti, O., Jasinskiene, N., James, A. A., Alphey, L., 2010. Female-specific flightless phenotype for mosquito control. Proc Nat'l Acad Sci USA 107, 4550-4554, doi:10.1073/pnas.1000251107.

Gillespie, J. H., 1998. Population genetics: a concise guide. The Johns Hopkins University Press, Baltimore and London.

Gong, P., Epton, M. J., Fu, G., Scaife, S., Hiscox, A., Condon, K. C., Condon, G. C., Morrison, N. I., Kelly, D. W., Dafa'alla, T., Coleman, P. G., Alphey, L., 2005. A dominant lethal genetic system for autocidal control of the Mediterranean fruitfly. Nature Biotechnology 23, 453-456, doi:10.1038/nbt1071.

Gould, F., Huang, Y., Legros, M., Lloyd, A. L., 2008. A Killer-Rescue system for selflimiting gene drive of anti-pathogen constructs. Proceedings of the Royal Society B: Biological Sciences 275, 2823-2829, doi:10.1098/rspb.2008.0846.

Gould, F., Anderson, A., Jones, A., Sumerford, D., Heckel, D. G., Lopez, J., Micinski, S., Leonard, R., Laster, M., 1997. Initial frequency of alleles for resistance to Bacillus 
thuringiensis toxins in field populations of Heliothis virescens. Proc Natl Acad Sci USA 94, 3519-3523, doi:10.1073/pnas.94.8.3519.

Hartley, C. J., Newcomb, R. D., Russell, R. J., Yong, C. G., Stevens, J. R., Yeates, D. K., La Salle, J., Oakeshott, J. G., 2006. Amplification of DNA from preserved specimens shows blowflies were preadapted for the rapid evolution of insecticide resistance. Proc Natl Acad Sci USA 103, 8757-8762, doi:10.1073/pnas.0509590103.

Horn, C., Wimmer, E., 2003. A transgene-based, embryo-specific lethality system for insect pest management. Nature Biotechnology 21, 64-70, doi:10.1038/nbt769.

Huang, F., Leonard, B. R., Andow, D. A., 2007. Sugarcane Borer (Lepidoptera: Crambidae) resistance to transgenic Bacillus thuringiensis maize. J Econ Entomol $100,164-171$.

Knipling, E., 1955. Possibilities of insect control or eradication through use of sexually sterile males. J. Econ. Entomol. 48, 459-462.

Mahon, R. J., Olsen, K. M., Downes, S., Addison, S., 2007. Frequency of Alleles Conferring Resistance to the Bt Toxins Cry1 Ac and Cry2Ab in Australian Populations of Helicoverpa armigera (Lepidoptera: Noctuidae). J Econ Entomol $100,1844-1853$.

Marrelli, M. T., Moreira, C. K., Kelly, D., Alphey, L., Jacobs-Lorena, M., 2006. Mosquito transgenesis: what is the fitness cost? Trends in Parasitology 22, 197-202, doi:10.1016/j.pt.2006.03.004. 
Nicholson, G. M., 2007. Fighting the global pest problem: Preface to the special Toxicon issue on insecticidal toxins and their potential for insect pest control. Toxicon 49, 413-422, doi:10.1016/j.toxicon.2006.11.028.

Phuc, H. K., Andreasen, M. H., Burton, R. S., Vass, C., Epton, M. J., Pape, G., Fu, G., Condon, K. C., Scaife, S., Donnelly, C. A., Coleman, P. G., White-Cooper, H., Alphey, L., 2007. Late-acting dominant lethal genetic systems and mosquito control. BMC Biology 5, 11, doi:10.1186/1741-7007-5-11.

Rendón, P., McInnis, D., Lance, D., Stewart, J., 2004. Medfly (Diptera:Tephritidae) genetic sexing: large-scale field comparison of males-only and bisexual sterile fly releases in Guatemala. J. Econ. Entomol. 97, 1547-1553.

Reynolds, A., 2007. Why EPA thinks resistance monitoring for Bt crops has been important and where do we go from here. Entomological Society of America Annual Meeting, San Diego, CA.

Schetelig, M. F., Horn, C., Handler, A. M., Wimmer, E. A., 2007. Development of an embryonic lethality system in Mediterranean fruit fly Ceratitis capitata. In: Vreysen, M., et al., Eds.), Area-wide control of insect pests: from research to field implementation. Springer, Dordrecht, The Netherlands, pp. 85-94.

Stainton, K. C., Condon, G. C., Fu, G., O’Connell, S., Alphey, L., unpublished data. Development of a Female-Lethal Genetic System in the Mexican Fruit Fly, Anastrepha ludens.

Stodola, T. J., Andow, D. A., Hyden, A. R., Hinton, J. L., Roark, J. J., Buschman, L. L., Porter, P., Cronholm, G. B., 2006. Frequency of resistance to Bacillus thuringiensis 
toxin Cryl Ab in southern United States corn belt population of European corn borer (Lepidoptera: Crambidae). J Econ Entomol 99, 502-507.

Tabashnik, B. E., Dennehy, T. J., Carrière, Y., 2005. Delayed resistance to transgenic cotton in pink bollworm. Proc Natl Acad Sci USA 102, 15389-15393, doi:10.1073/pnas.0507857102

Thomas, D. D., Donnelly, C. A., Wood, R. J., Alphey, L. S., 2000. Insect population control using a dominant, repressible, lethal genetic system. Science 287, 24742476.

Yakob, L., Alphey, L., Bonsall, M. B., 2008. Aedes aegypti control: the concomitant role of competition, space and transgenic technologies. Journal of Applied Ecology 45, 1258-1265, doi:10.1111/j.1365-2664.2008.01498.x. 


\section{Figure Legends}

\section{Figure 1}

\section{Allele frequency evolution with complete, dominant, no-cost resistance}

The graphs show the frequency over time in naturally emerging adults of the $R$ allele (a \& b) and the $L$ allele (c \& d), for no release of insects (dotted lines, a $\&$ b), bisex-lethal (BL) RIDL release (solid lines) and female-lethal (FL) RIDL release (dashed lines). The release ratio is 10:1 $(d=10)$, i.e. ten RIDL males per male in the wild. The $R$ allele has no fitness costs: relative fitness $\psi_{S R}=\psi_{R R}=1$. Resistance is dominant and complete: susceptibility $\gamma_{S R}=\gamma_{R R}=0$. Initial $R$ allele frequency is $(\mathrm{a} \& \mathrm{c}) p_{0}=0.1$ or $(\mathrm{b} \& \mathrm{~d}) p_{0}=0.001$. The $L$ construct is fully lethal to targets, with no other fitness costs: $\varepsilon_{\text {target }}=1$ and $\varepsilon_{\text {non-target }}=0$. 


\section{Figure 2}

\section{The effect of release ratio on allele frequency evolution}

The graphs show the frequency over time of the $R$ allele (a $\&$ b) and the $L$ allele (c $\& \mathrm{~d}$ ), for bisex-lethal RIDL release (a \& c) or female-lethal RIDL release (b \& d). The release ratios are 1:2 $(d=0.5$, solid lines), 1:1 ( $d=1$, dashed lines), 10:1 (as Fig. $1, d=10$, dotted lines) and 1000:1 ( $d=1000$, dash-dot lines). Genetic parameters of $R$ and $L$ alleles are as in Fig. 1 (complete, dominant, no-cost resistance). Initial $R$ allele frequency $p_{0}=0.001$. 


\section{Figure 3}

\section{Allele frequency evolution with complete, recessive, no-cost resistance}

The graphs show the frequency over time of the $R$ allele (a) and the $L$ allele (b), for no release of insects (dotted line, obscured behind solid line in panel a), bisex-lethal RIDL release (solid lines) and female-lethal RIDL release (dashed lines). The release ratio is 10:1 $(d=10)$. The $R$ allele has no fitness costs: relative fitness $\psi_{S R}=\psi_{R R}=1$. Resistance is recessive $\gamma_{S R}=\gamma_{S S}=1$ and complete: susceptibility $\gamma_{R R}=0$. Initial $R$ allele frequency $p_{0}=0.001$. The $L$ construct is fully-lethal to targets, with no other fitness costs: $\varepsilon_{\text {target }}=1$ and $\varepsilon_{\text {non-target }}=0$. The $L$ allele equilibrium frequency with $\mathrm{FL}$ is $l^{*}=\frac{1 / 2 \times 10}{1+10}=0.4545(\mathrm{Eq}$. 12). Note the y-axis in (a) is scaled 0 to approximately $p_{0}=0.001$ (not 0 to 1 ). 


\section{Figure 4}

\section{The effect of varying susceptibility on allele frequency evolution}

The graphs show the frequency over time of the $R$ allele (a $\& \mathrm{~b}$ ) and the $L$ allele (c $\& \mathrm{~d}$ ), for bisex-lethal RIDL release (a \& c) or female-lethal RIDL release (b \& d). The release ratio is 10:1 $(d=10)$. Initial $R$ allele frequency $p_{0}=0.001$. The $R$ allele has no fitness costs: relative fitness $\psi_{S R}=\psi_{R R}=1$. Resistance is dominant: susceptibility $\gamma_{S R}=\gamma_{R R}$ taking values 0 (complete resistance, solid lines), 0.3 (dashed lines), 0.5 (dotted lines), 0.7 (dashdot lines) or 0.9 (circles). The $L$ construct is fully-lethal to targets, with no other fitness costs: $\varepsilon_{\text {target }}=1$ and $\varepsilon_{\text {non-target }}=0$. 


\section{Figure 5}

\section{The effect of susceptibility to the RIDL construct on equilibrium allele frequencies}

The graphs show the equilibrium frequencies of the $R$ allele (a \& b) and the $L$ allele (c \& d), for bisex-lethal RIDL release (a \& c) or female-lethal RIDL release (b \& d) against susceptibility to the RIDL construct ranging over all permitted values $\left(0 \leq \gamma_{R R} \leq 0 \gamma_{S R} \leq 1\right)$. The release ratio is 10:1 $(d=10)$. Initial $R$ allele frequency $p_{0}=0.01$. The $R$ allele has no fitness costs: relative fitness $\psi_{S R}=\psi_{R R}=1$. The $L$ construct is fully-lethal to targets, with no other fitness costs: $\varepsilon_{\text {target }}=1$ and $\varepsilon_{\text {non-target }}=0$. 


\section{Figure 6}

\section{The effect of fitness costs of resistance on equilibrium allele frequencies}

The graphs are as for Fig. 5 b \& d, except for fitness costs of resistance. They show the equilibrium frequencies of the $R$ allele (a \& b) and the $L$ allele (c \& d), for female-lethal RIDL release against susceptibility to the RIDL construct ranging over all permitted values $\left(0 \leq \gamma_{R R} \leq 0 \gamma_{S R} \leq 1\right)$. The $L$ construct is fully-lethal to targets, with no other fitness costs:

$\varepsilon_{\text {target }}=1$ and $\varepsilon_{\text {non-target }}=0$. The release ratio is 10:1 $(d=10)$. Initial $R$ allele frequency $p_{0}=0.01$. The $R$ allele has fitness costs: relative fitness (a \& c) $\psi_{S R}=0.9, \psi_{R R}=0.7$, "fit resistance" or (b \& d) $\psi_{S R}=0.2, \psi_{R R}=0.1$, "costly resistance". 


\section{Figure 7}

\section{Population dynamics with density-dependent mortality}

The graphs show the relative population size $N$ over time, for no release of insects (circles), bisex-lethal RIDL release (solid lines) and female-lethal RIDL release (dashed lines). For comparison, totals with no resistance present are shown for bisex-lethal (dash-dot lines) and female-lethal (dotted) RIDL release. Initial $R$ allele frequency $p_{0}$ is 0.01 . The $L$ construct is fully lethal to targets, with no other fitness costs: $\varepsilon_{\text {target }}=1$ and $\varepsilon_{\text {non-target }}=0$. Progeny per female (a \& c) $2 R_{0}=15$ or (b) $2 R_{0}=6$, with release ratio (a \& c) $20: 1(d=20)$ or (b) 10:1 ( $d=10)$. The $R$ allele has (a) minor fitness costs, with relative fitness $\psi_{S R}=0.95, \psi_{R R}=0.85$, or $(\mathrm{b} \& \mathrm{c})$ no $\operatorname{costs} \psi_{S R}=\psi_{R R}=1$. Resistance is (a) modest, with susceptibility $\gamma_{S R}=0.9, \gamma_{R R}=0.7$, or (b \& c) strong, susceptibility $\gamma_{S R}=0.2, \gamma_{R R}=0.1$. The corresponding equilibrium allele frequencies are (a) BL: $p^{*}=0.5054, l^{*}=0.3312$, $\mathrm{FL}: p^{*}=0.2412, l^{*}=0.6906,(\mathrm{~b}) \mathrm{BL}: p^{*}=0.5288, l^{*}=1, \mathrm{FL}: p^{*}=0.3320, l^{*}=1,(\mathrm{c})$ $\mathrm{BL}: p^{*}=0.5151, l^{*}=1, \mathrm{FL}: p^{*}=0.3215, l^{*}=1$. 


\section{Tables}

Table 1

\section{Parameters and symbols}

\section{Symbol Parameter}

p frequency of resistant $R$ allele in current adult generation

$q \quad$ frequency of susceptible $S$ allele

$p_{0} \quad$ initial $R$ allele frequency

$p^{*} \quad$ equilibrium $R$ allele frequency

$l \quad$ frequency of the RIDL genetic construct $L$

$l_{0} \quad$ initial $L$ allele frequency

$l^{*} \quad$ equilibrium $L$ allele frequency

$i \quad$ genotype at $S / R$ locus

$j \quad$ genotype at locus of construct insertion ( $\mathrm{L}$ allele), or wild

type absence of the construct (w allele)

$k$ genotype for effect of RIDL construct:

target (both sexes for BL, females only for FL)

non-target (males only for FL)

$\psi_{i} \quad$ relative fitness of larvae of genotype $i$

$\varepsilon_{k} \quad$ fitness penalty of RIDL construct

$\gamma_{i} \quad$ susceptibility to the RIDL construct (scaling factor applied to fitness penalty $\varepsilon$ )

\section{Constraints}

$0 \leq p \leq 1$

$0 \leq q \leq 1, p+q=1$

$0<p_{0}<1$

$0 \leq l \leq 1$

$0 \leq l_{0}<1$

$S S, S R$ or $R R$

$w w, L w$ or $L L$

target or non-target

$0 \leq \psi_{R R} \leq \psi_{S R} \leq \psi_{S S}=1$

$0 \leq \varepsilon_{k} \leq 1$

$0 \leq \gamma_{R R} \leq \gamma_{S R} \leq \gamma_{S S}=1$,

$\gamma_{R R} \neq \gamma_{S S}$ 
$\eta_{j} \quad$ number of copies of the RIDL construct

$\Omega_{i j k} \quad$ relative fitness of larvae of genotype $i, j, k$

$d \quad$ release ratio of released RIDL males to naturally emerging adult males in the wild (of any genotype)

$R_{0} \quad$ average number of female progeny produced per adult female that survive to adulthood in a density-independent population

$N_{t} \quad$ population size of mature adults at generation $t$ relative to the initial pest density

$F_{t} \quad$ relative population size of mature females at generation $t$

$\sigma_{t} \quad$ simulated proportion of all offspring that survive to maturity

$\sigma_{t}^{(F)} \quad$ simulated proportion of female offspring that survive to
0 for $w w, 1$ for $L w$ or 2

for $L L$

(see Eq. 2)

$d>0$

$R_{0}>0$

$N_{0}=1$

(see Eqs. 4 or 8 )

(see Eqs. 5 or 9) maturity 


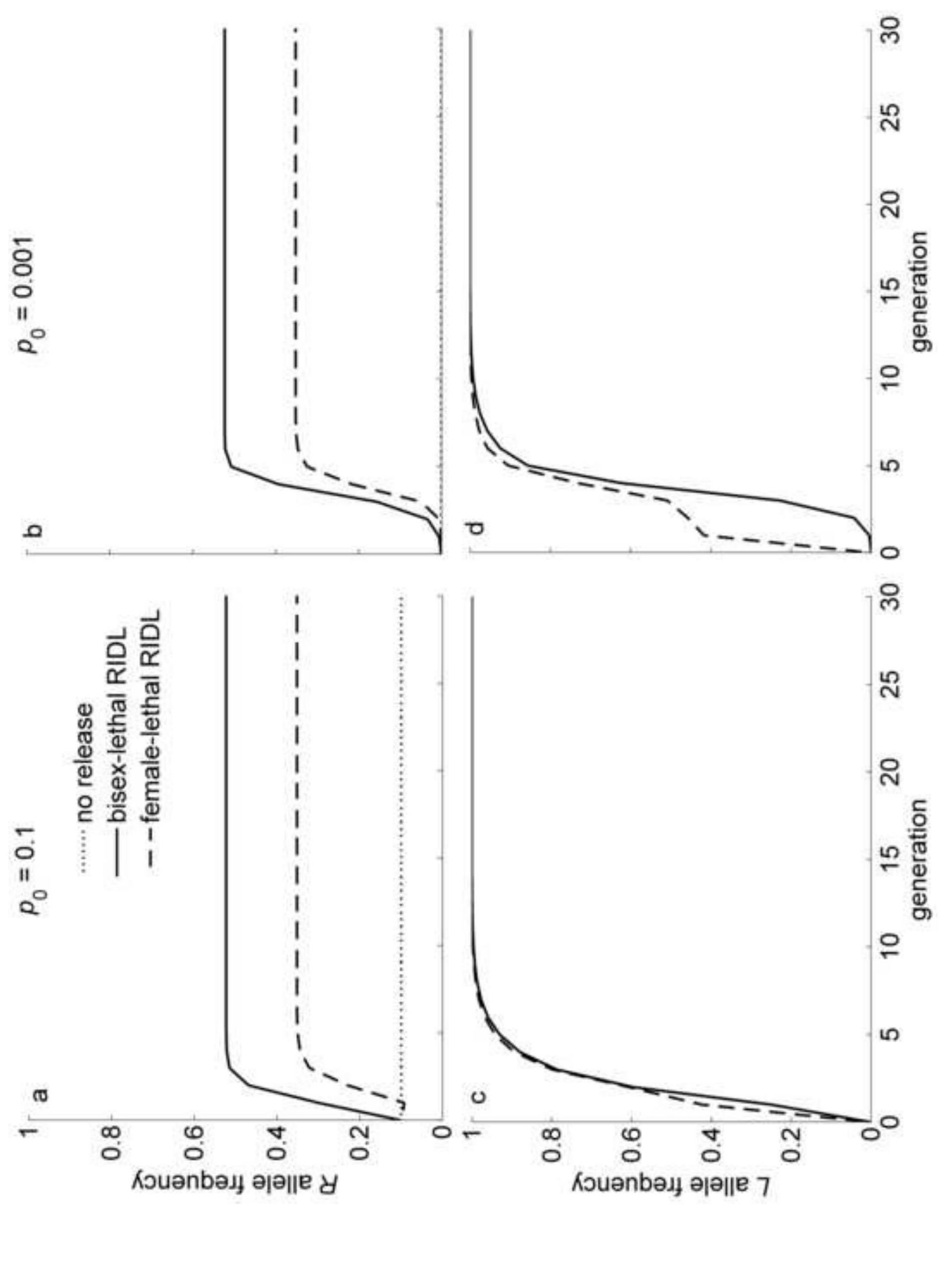




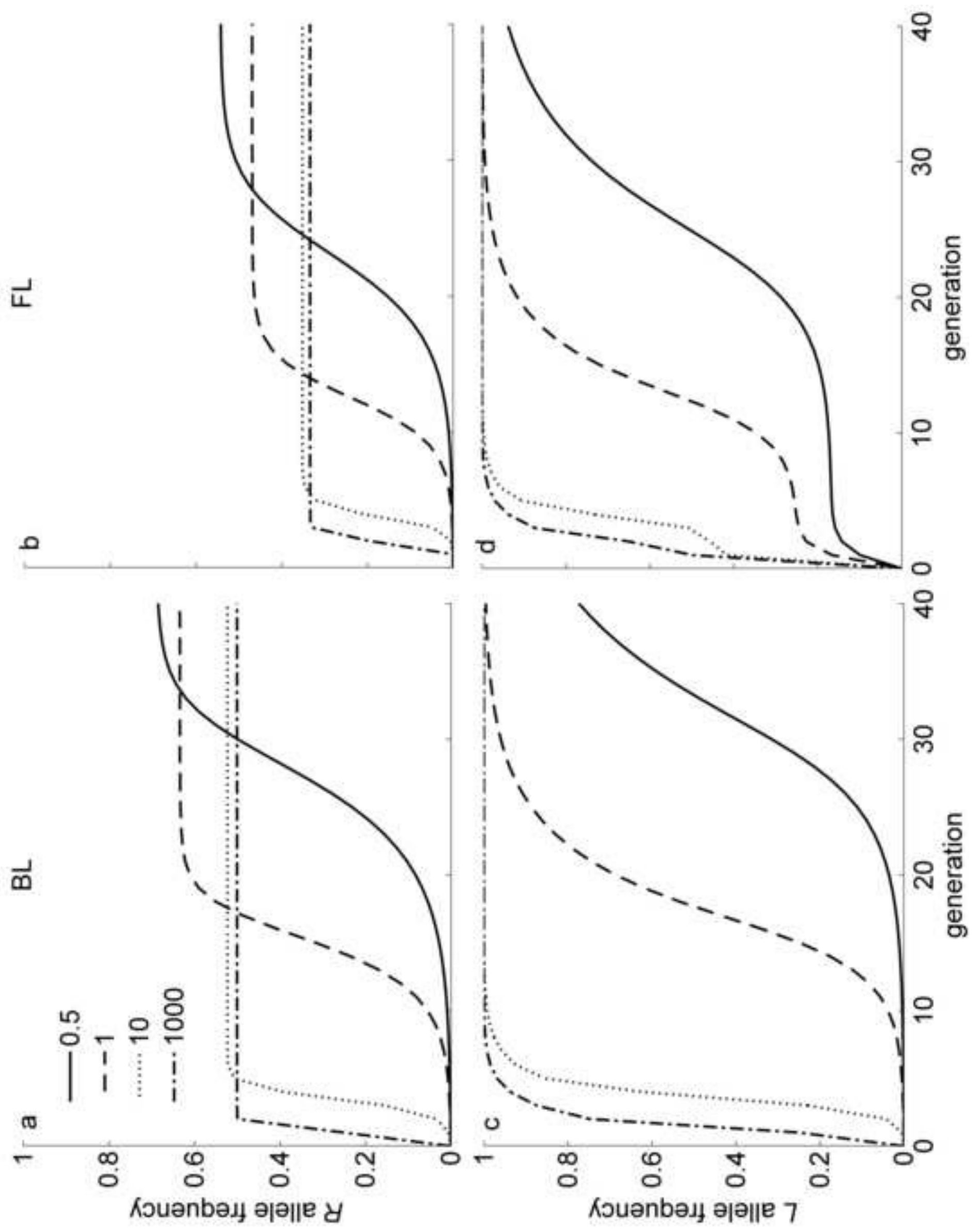

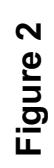



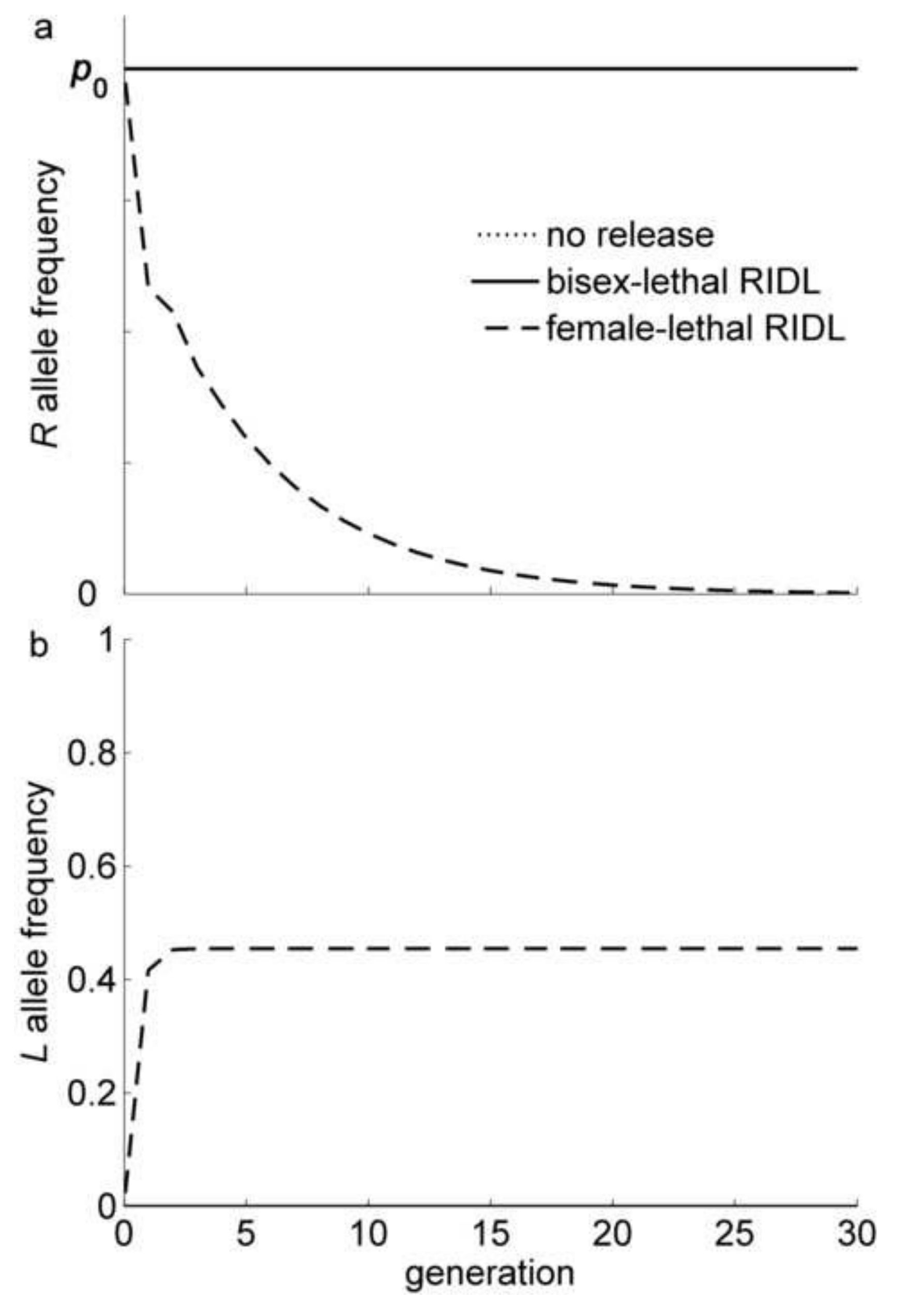

b 1 


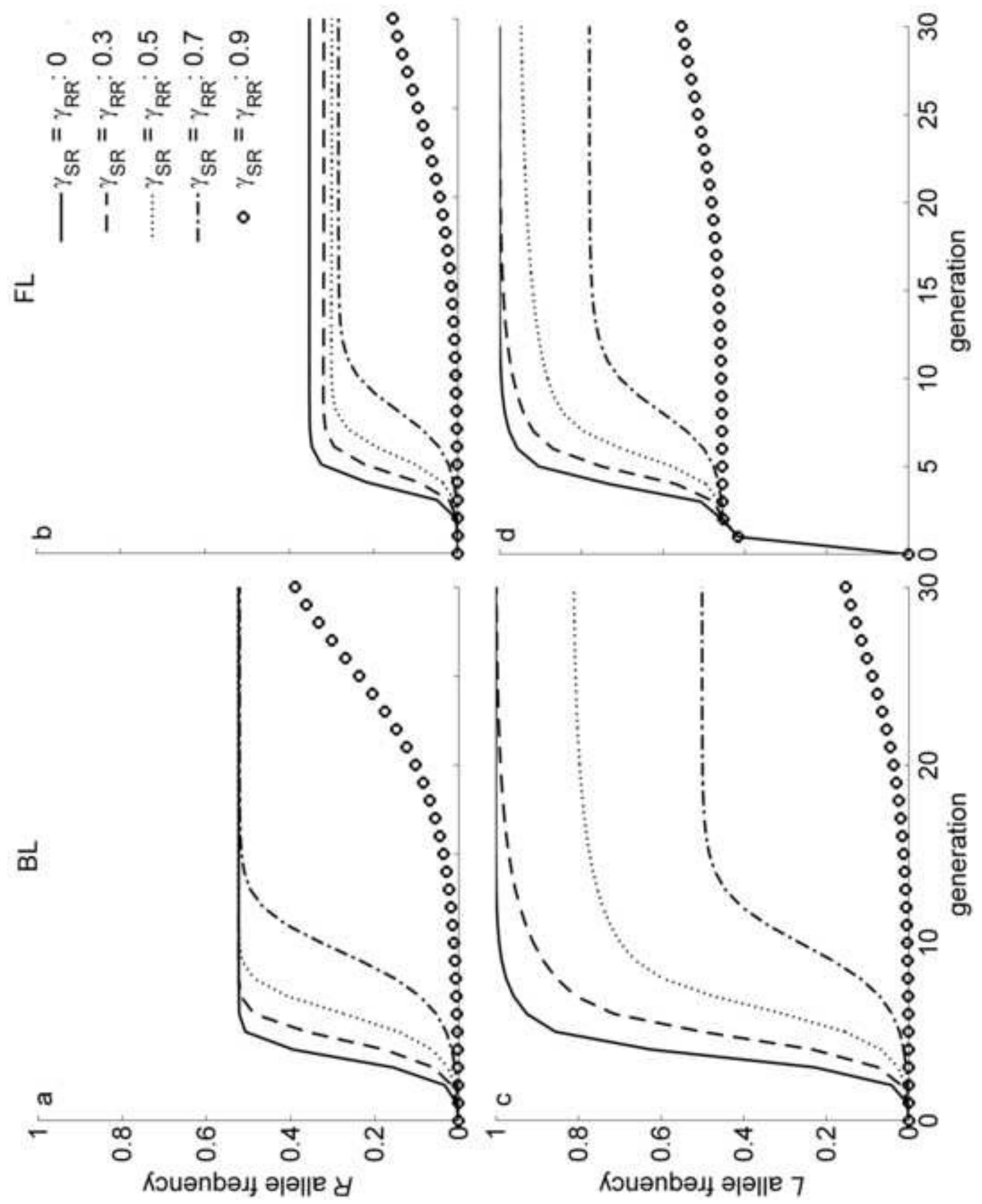




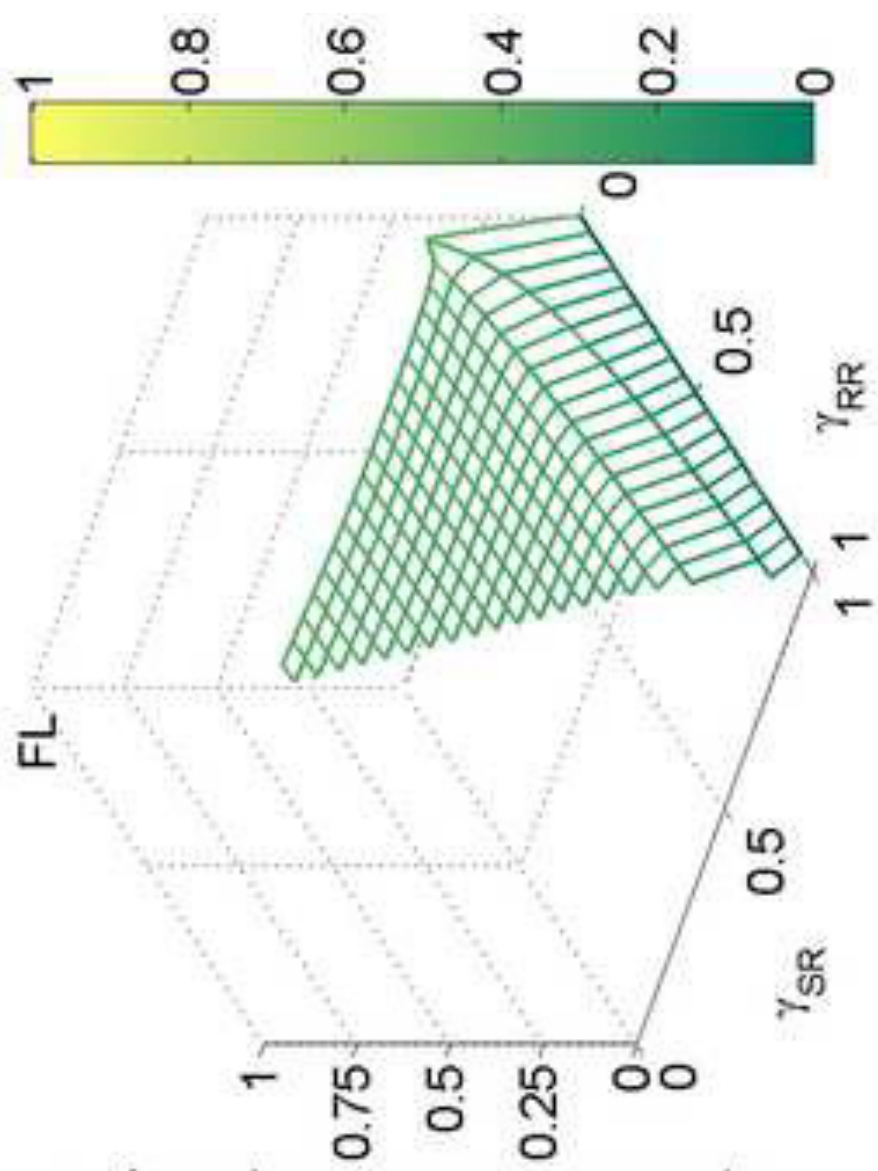

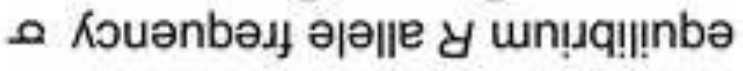

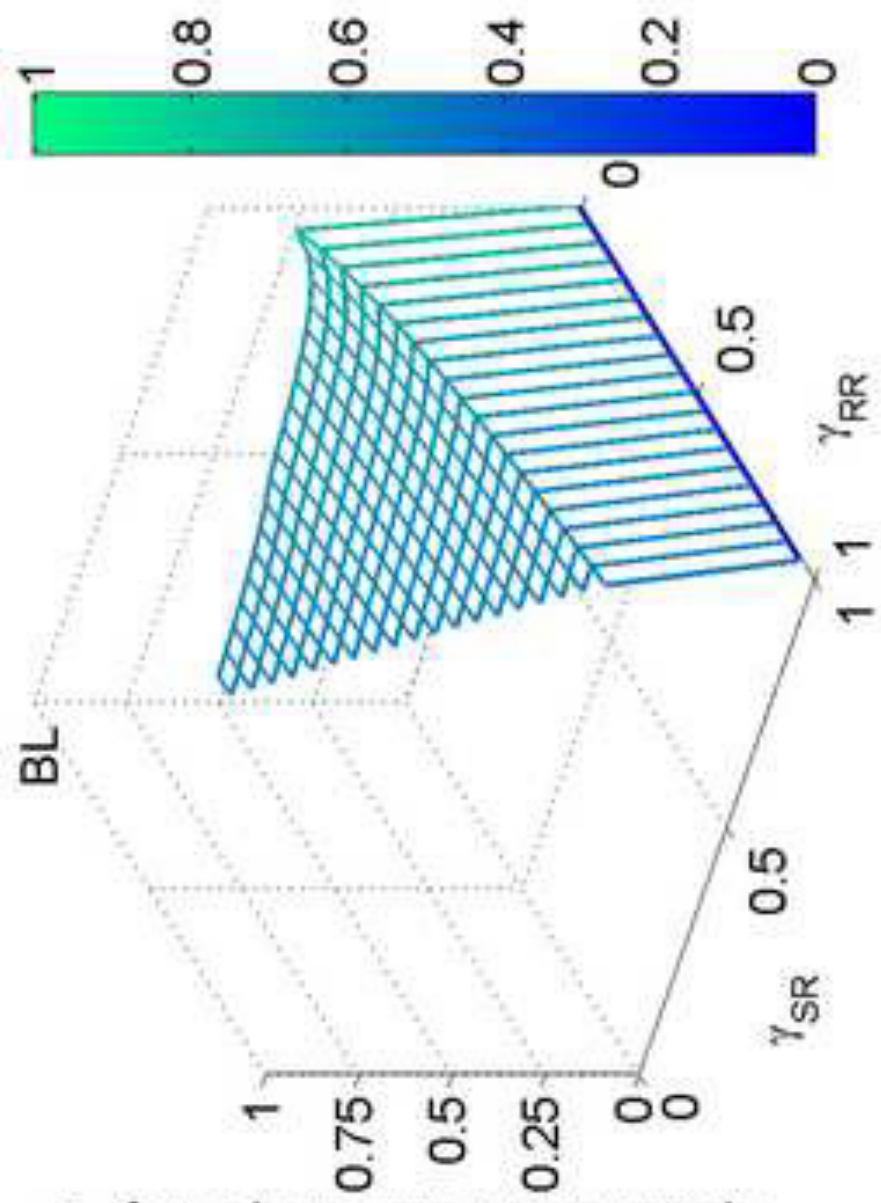

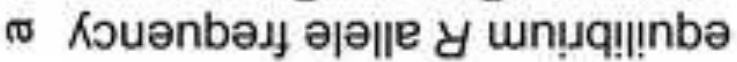

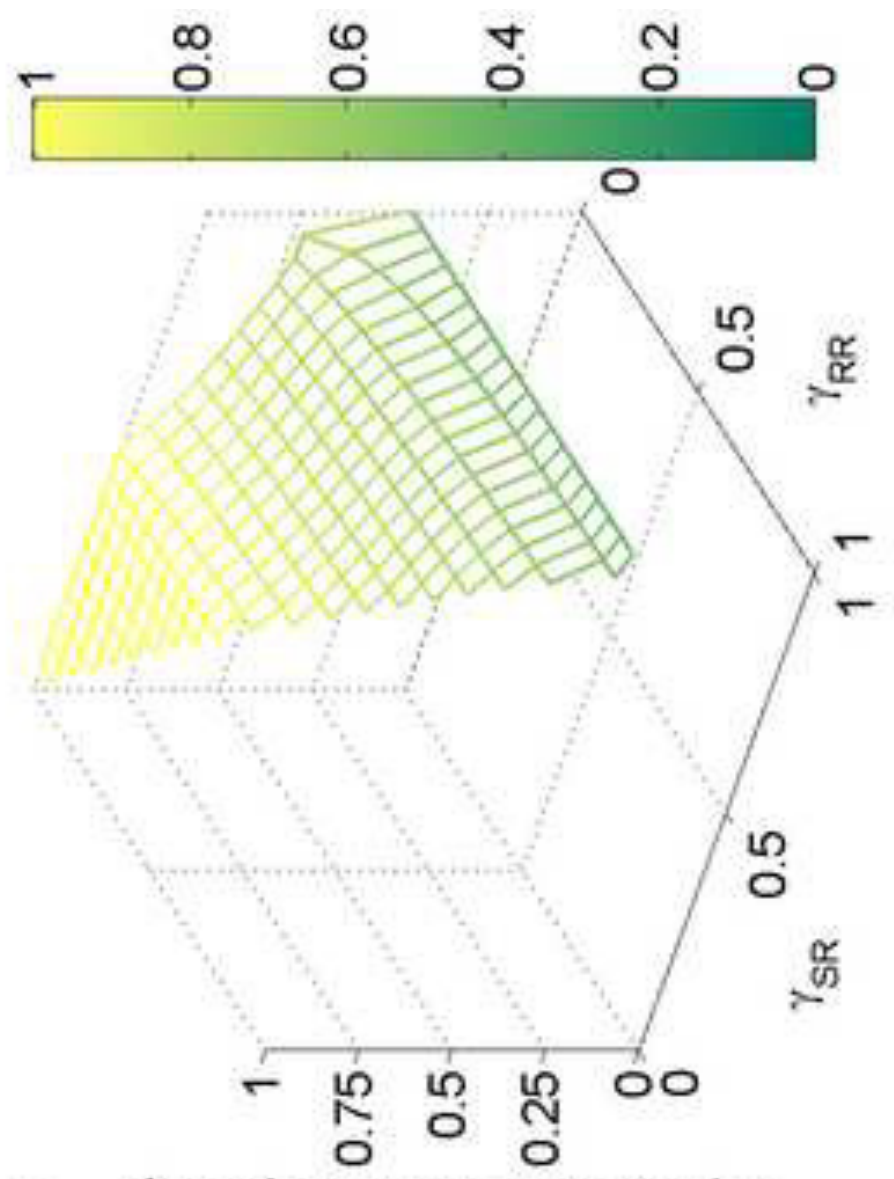

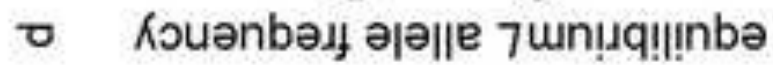

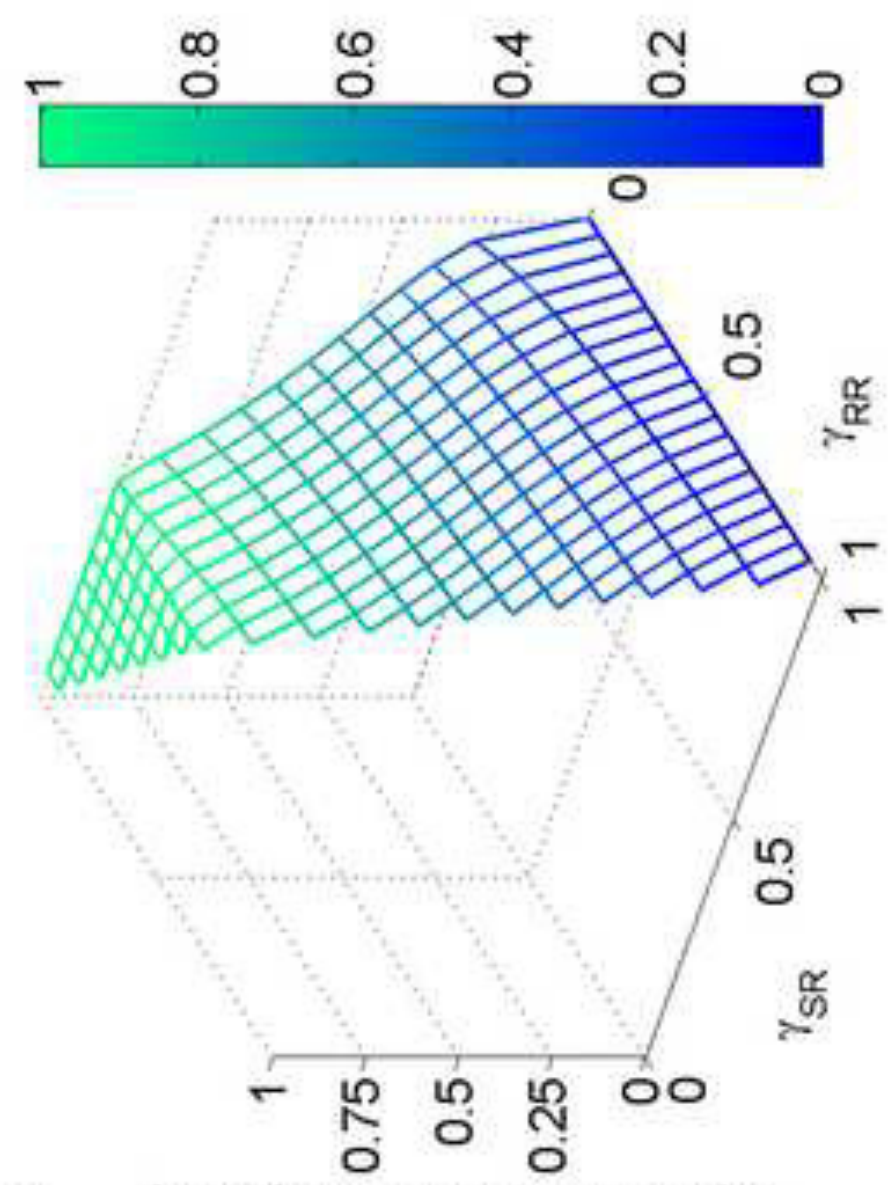

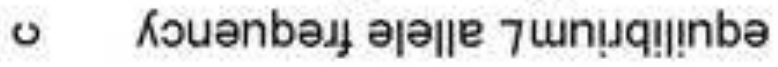



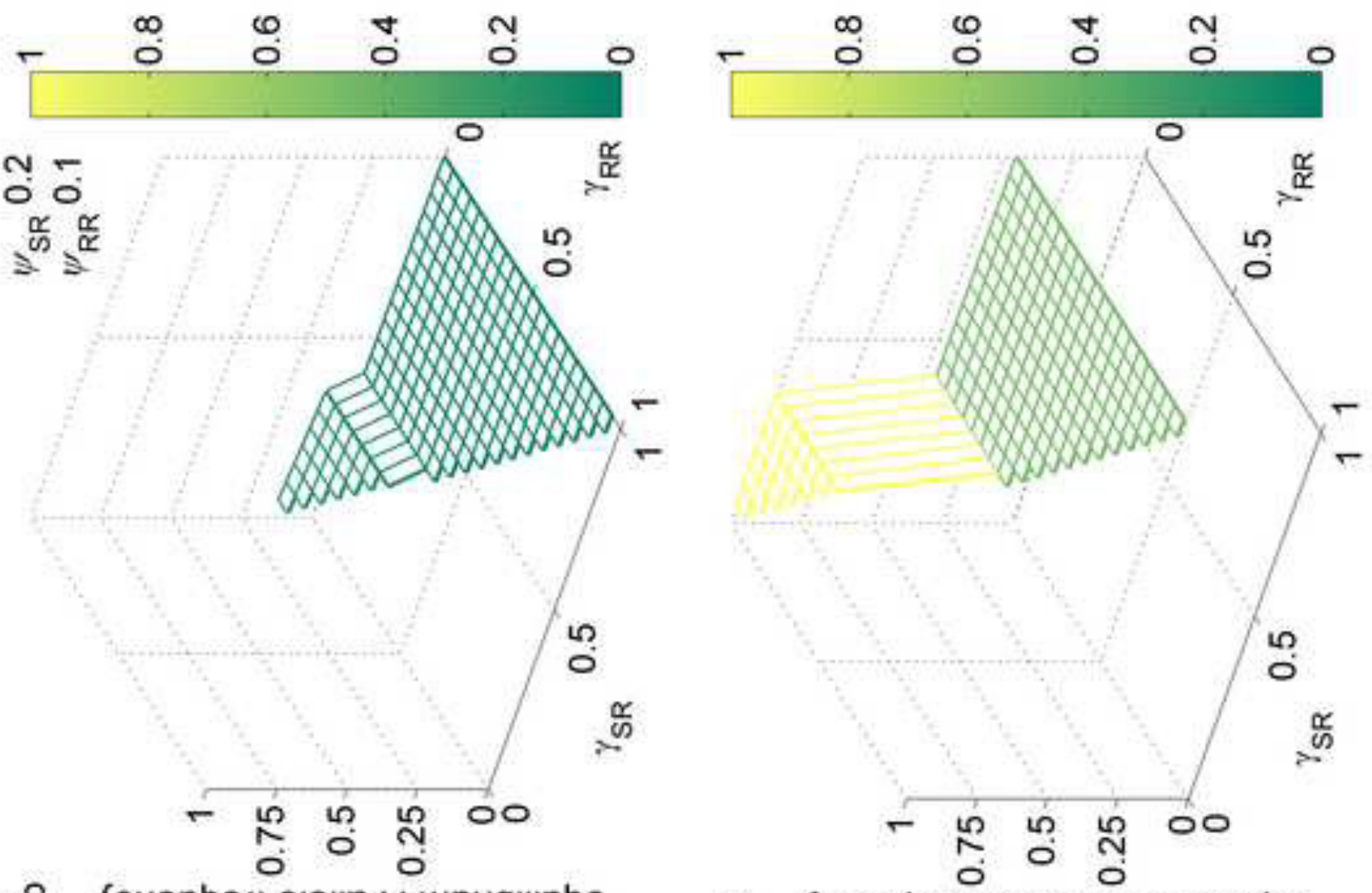

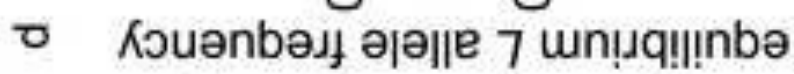

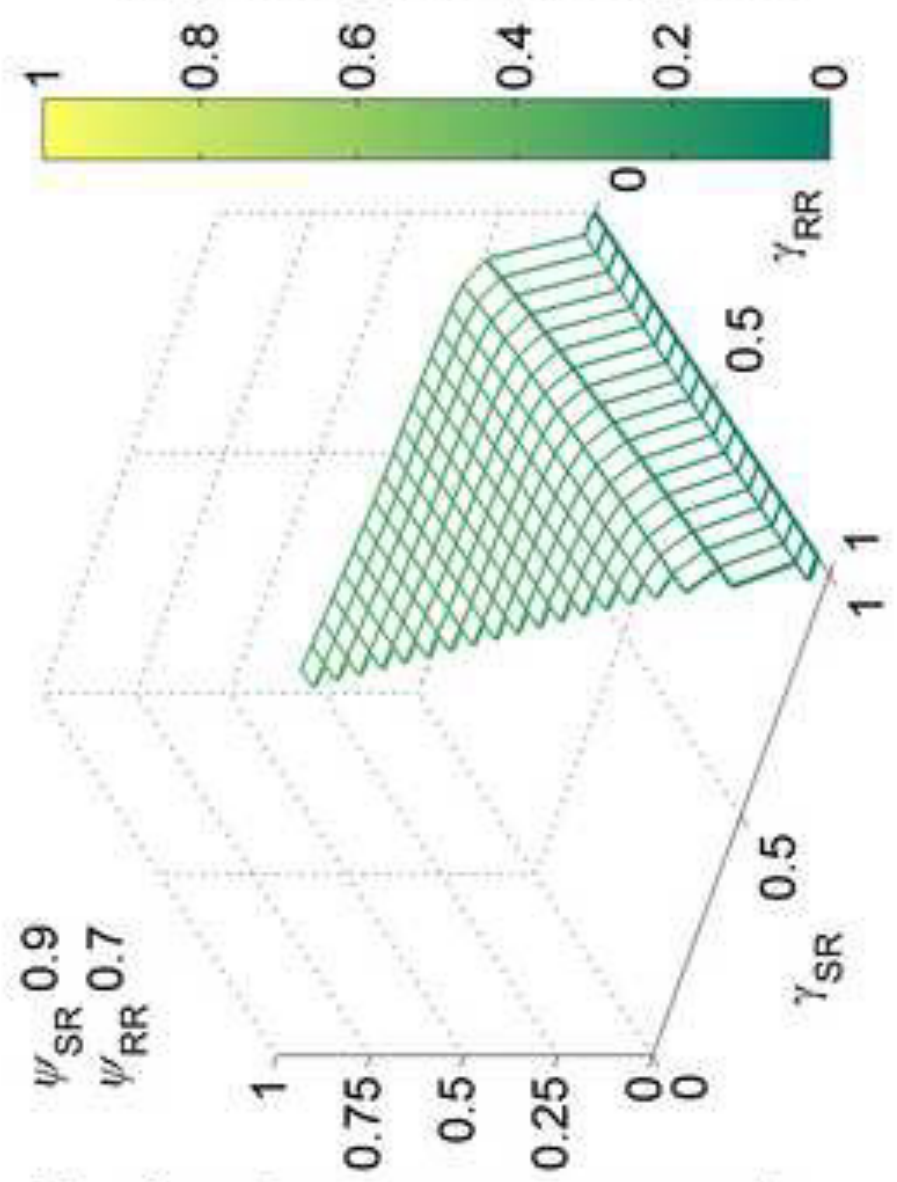

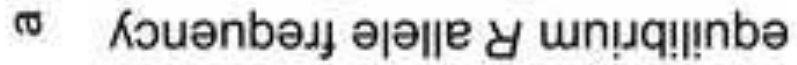

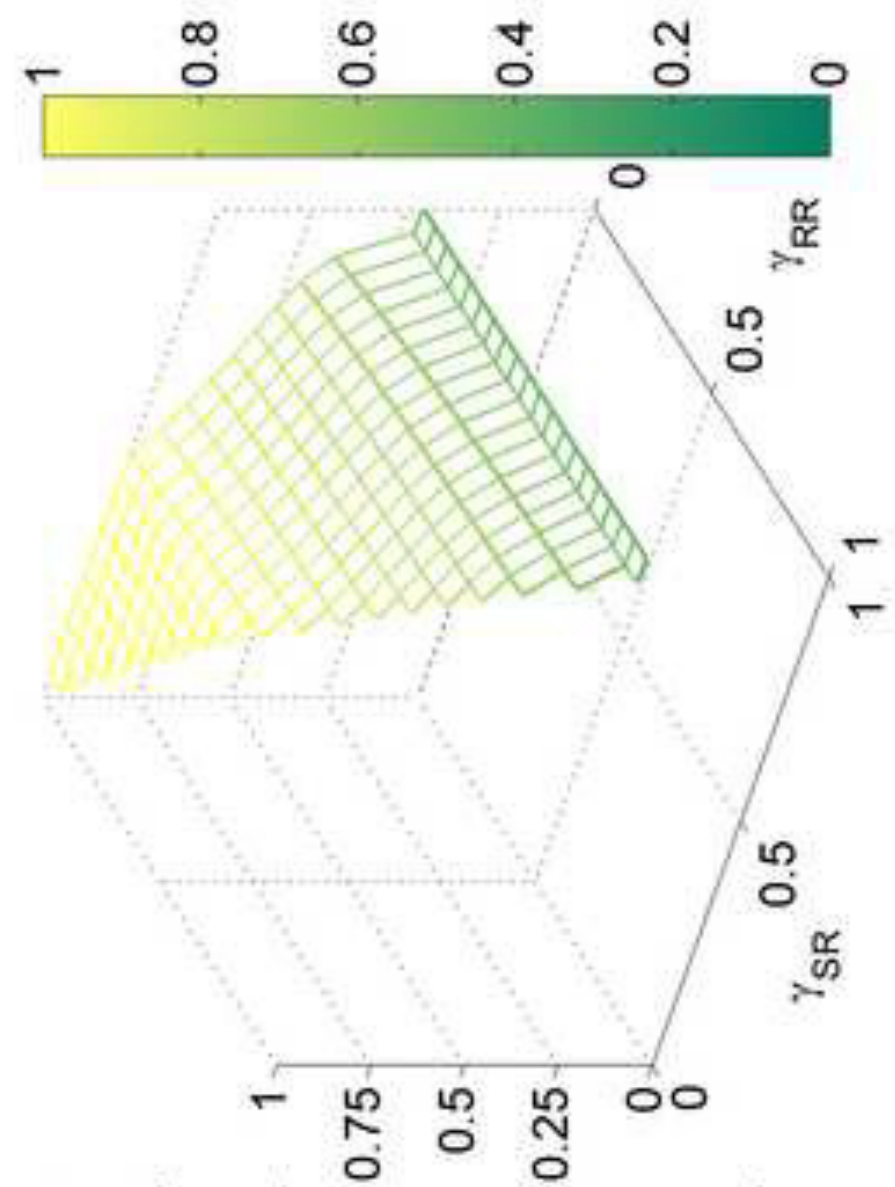

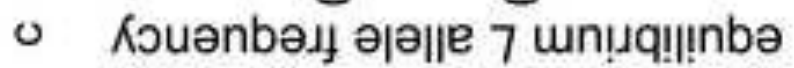



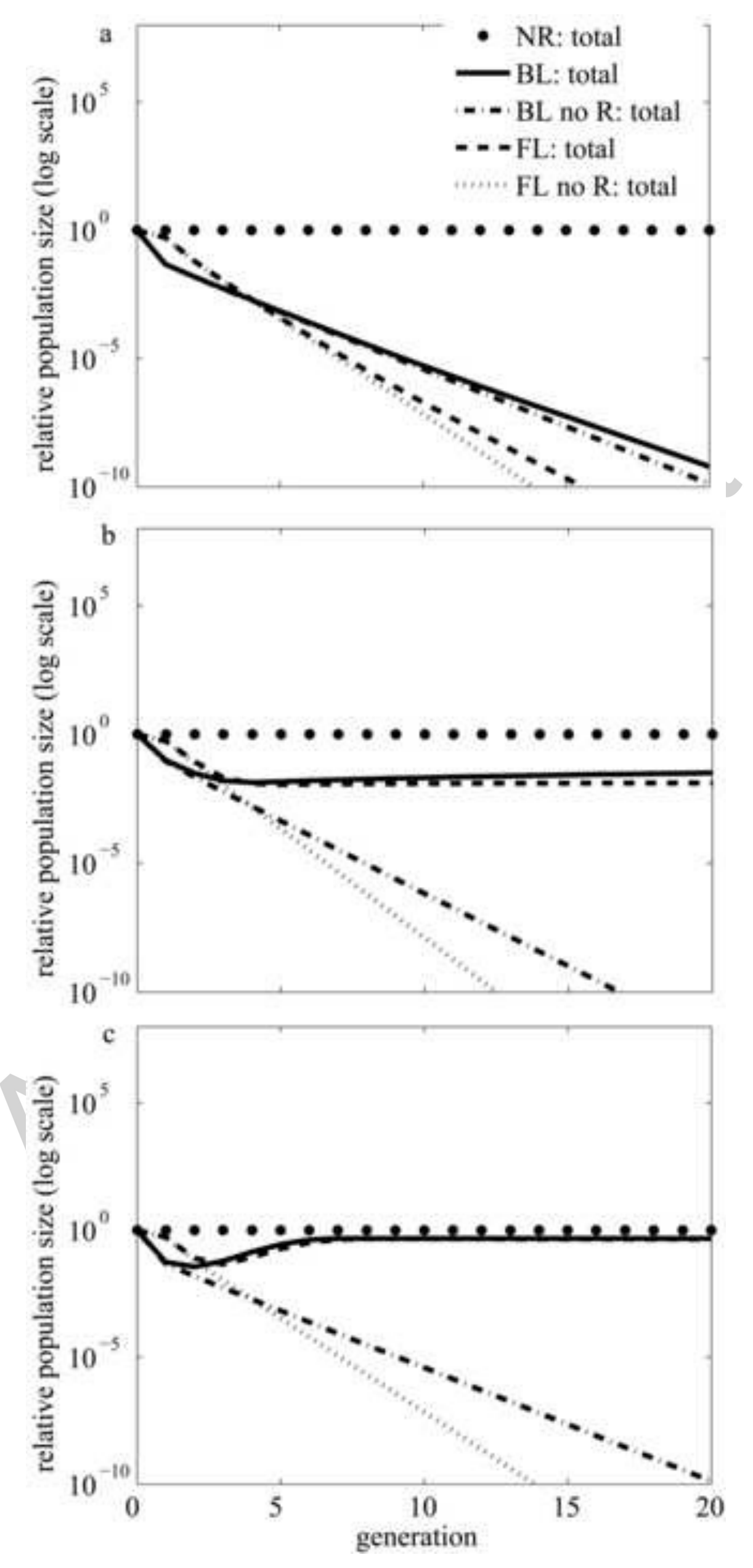

Figure 7

- BL: total

- - BL no R: total

FL no R: total 


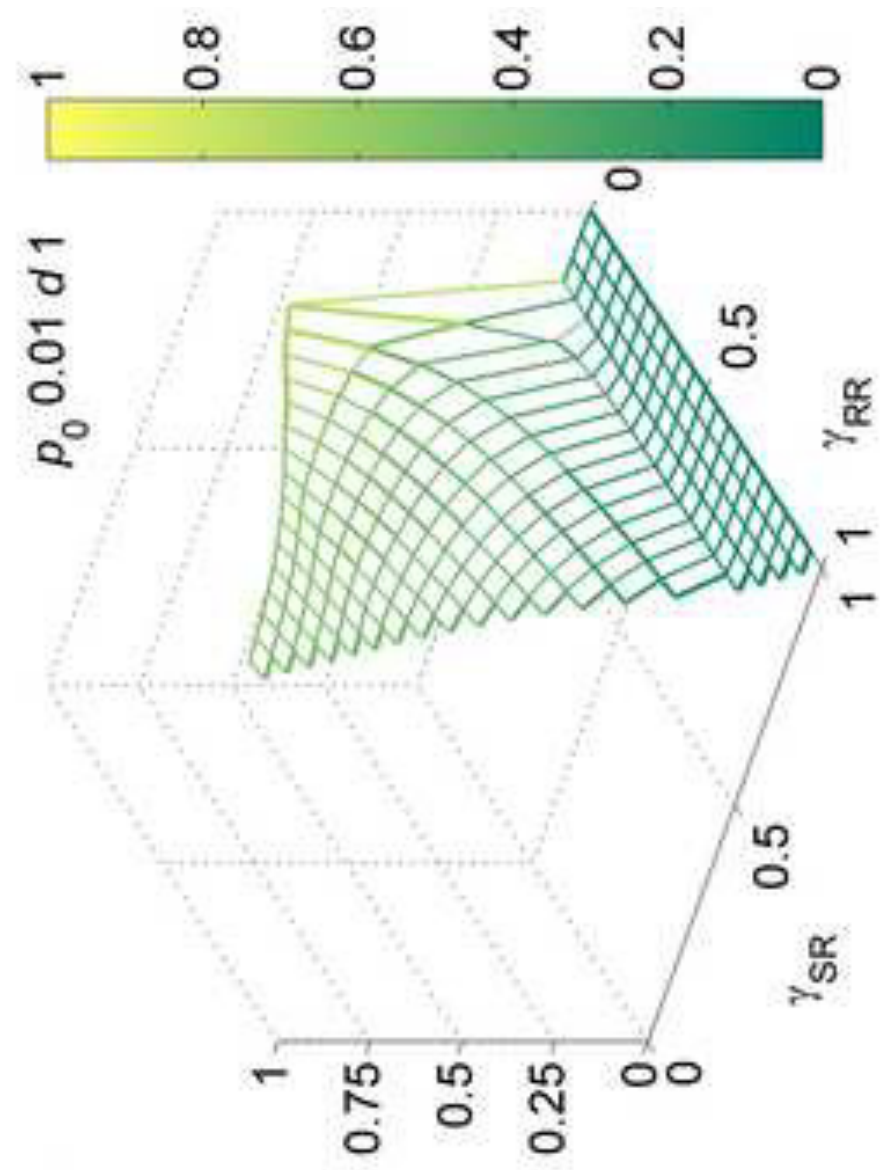

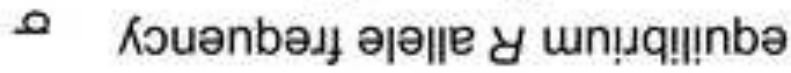

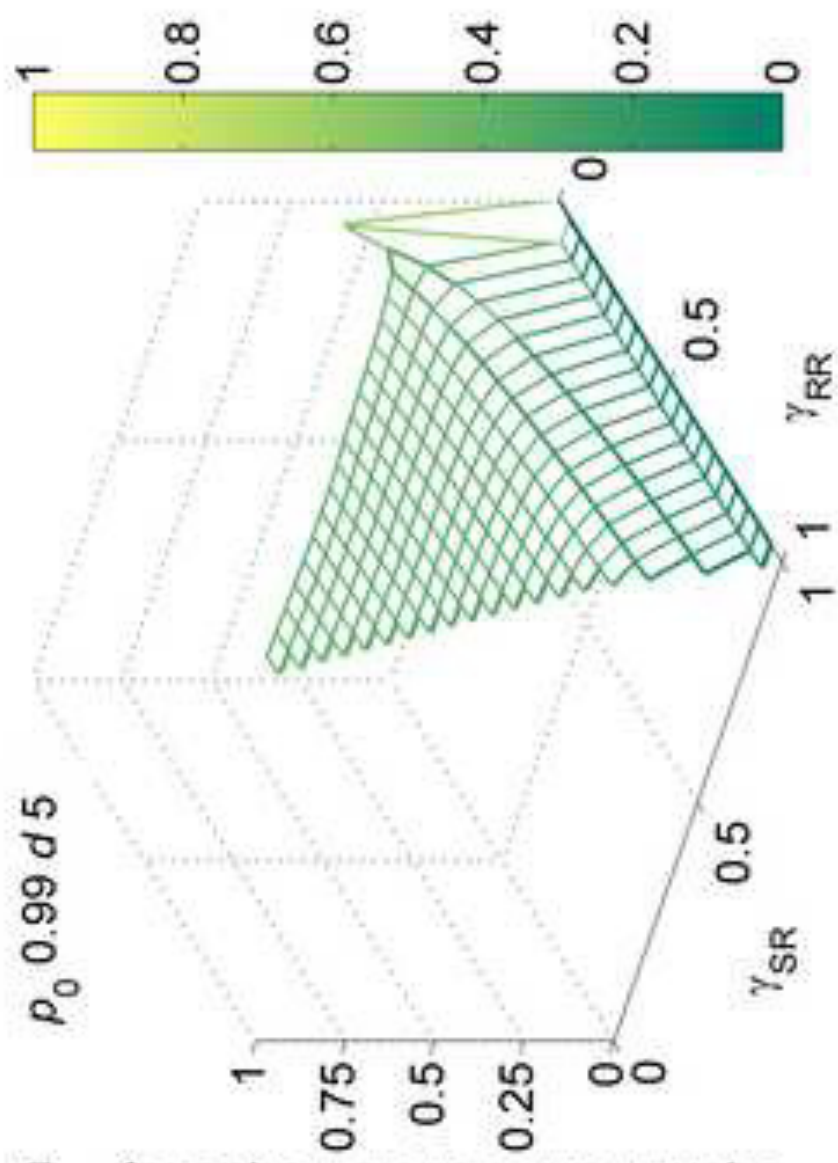

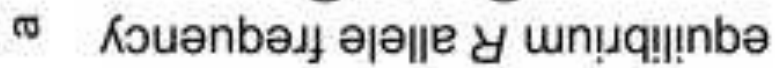

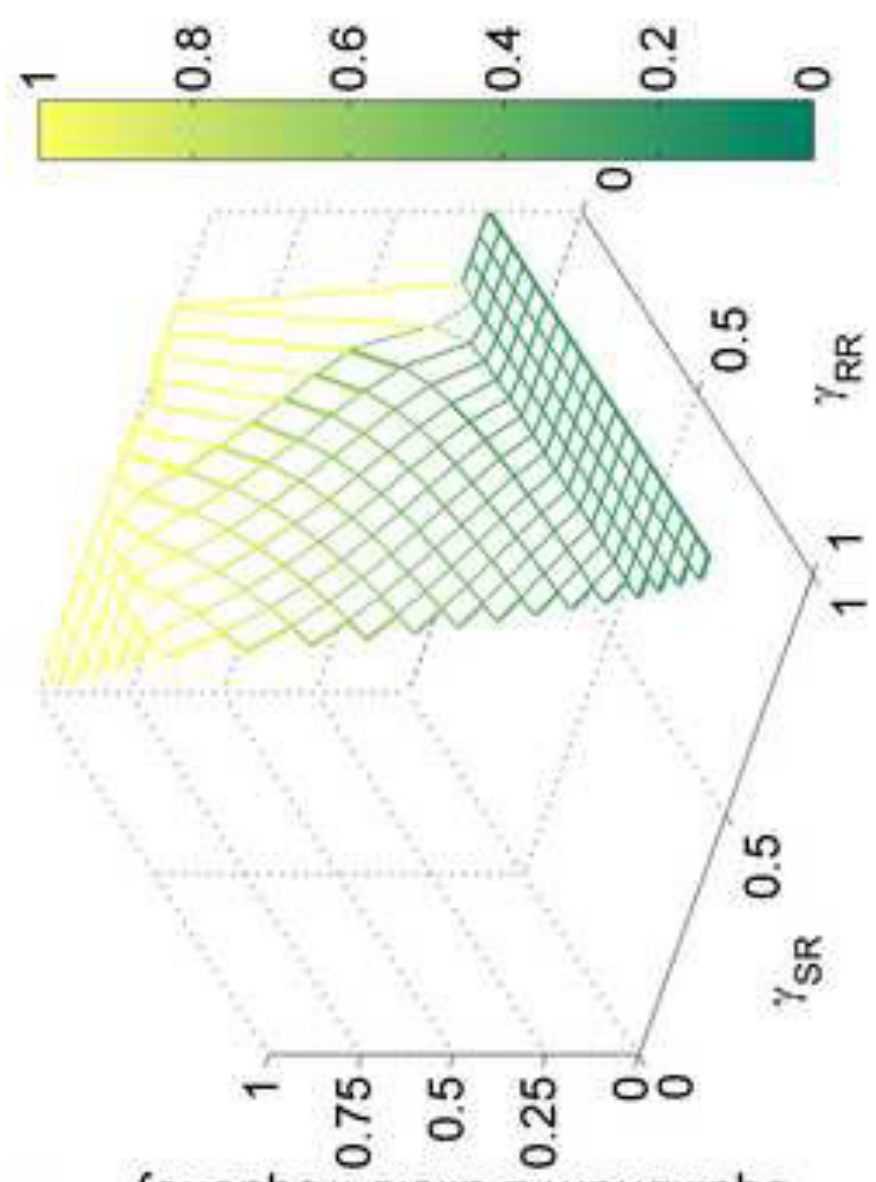

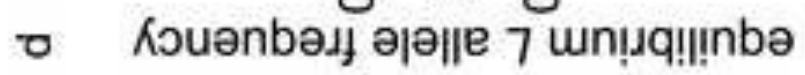
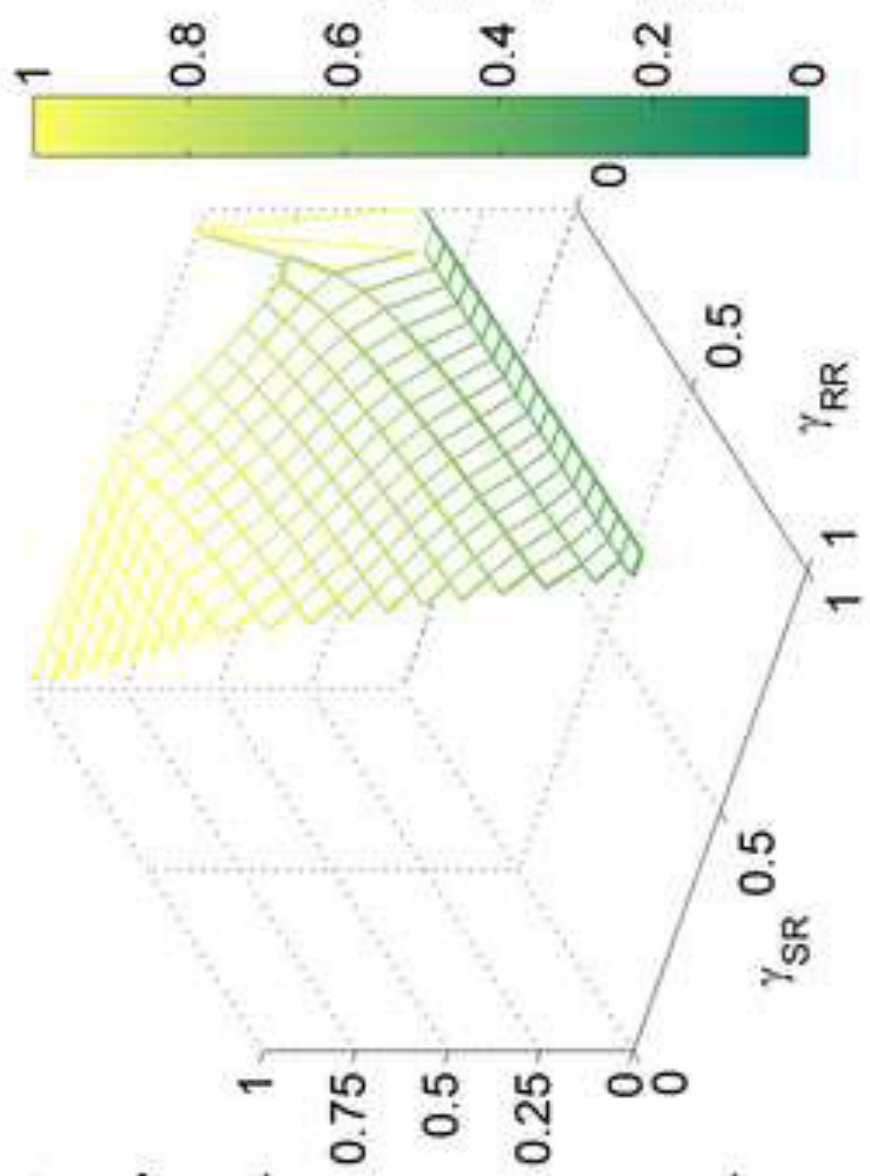

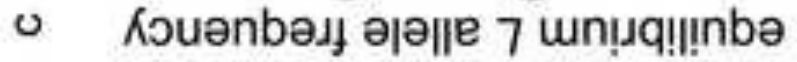



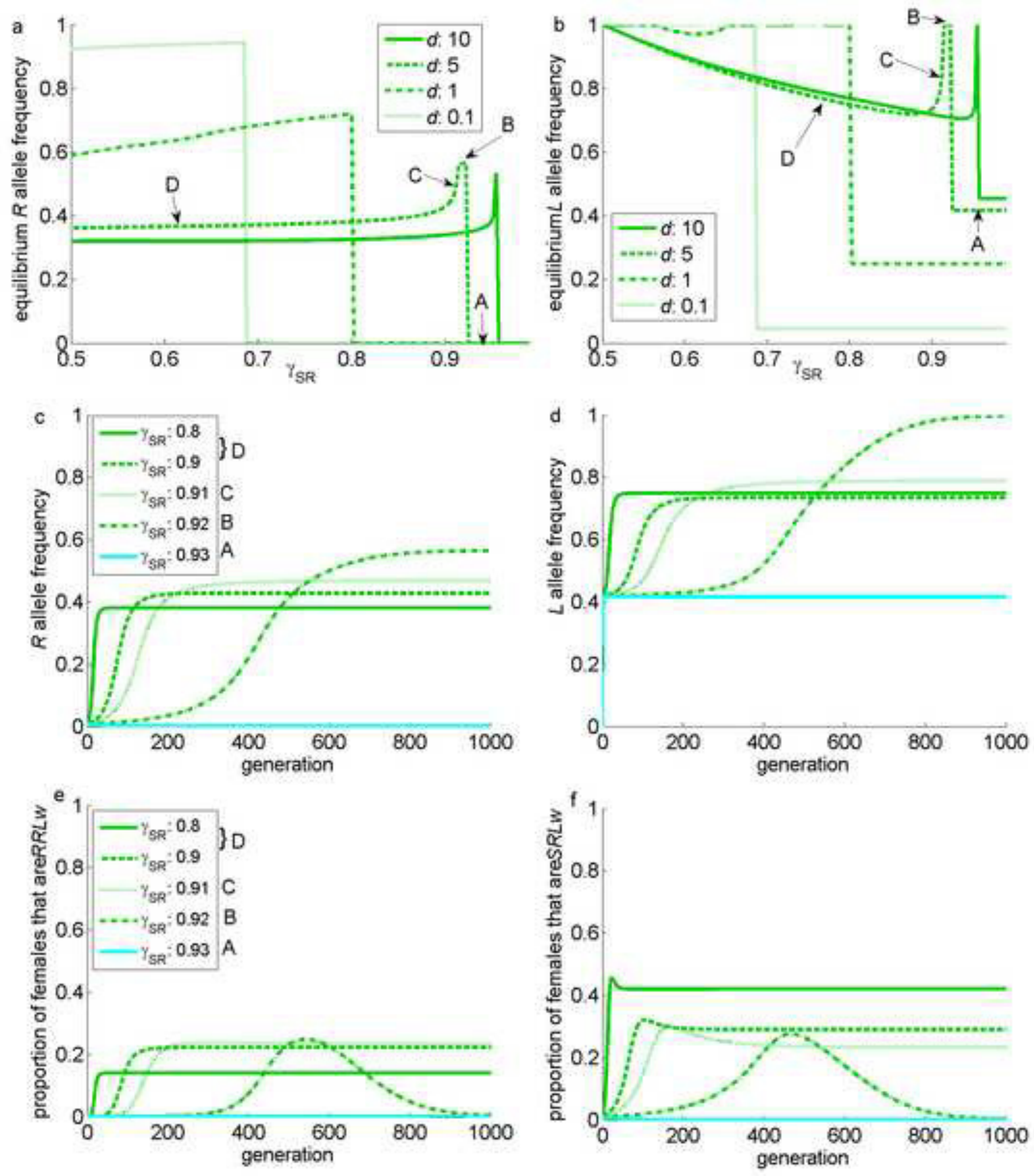

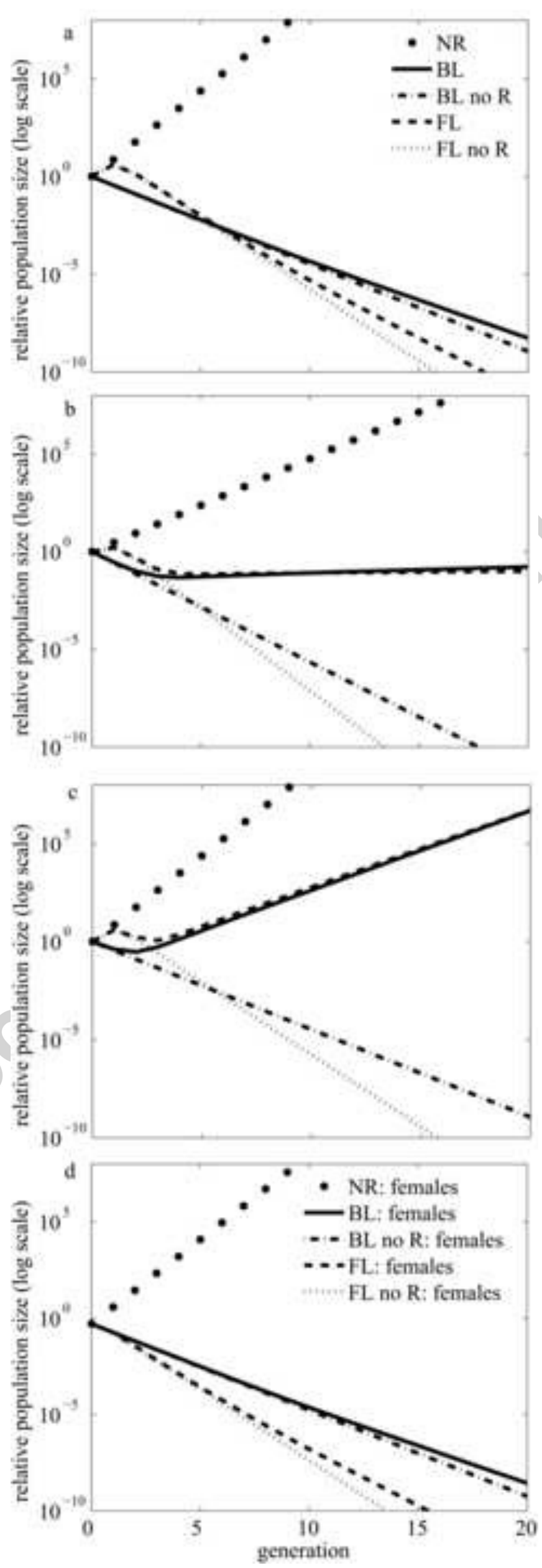


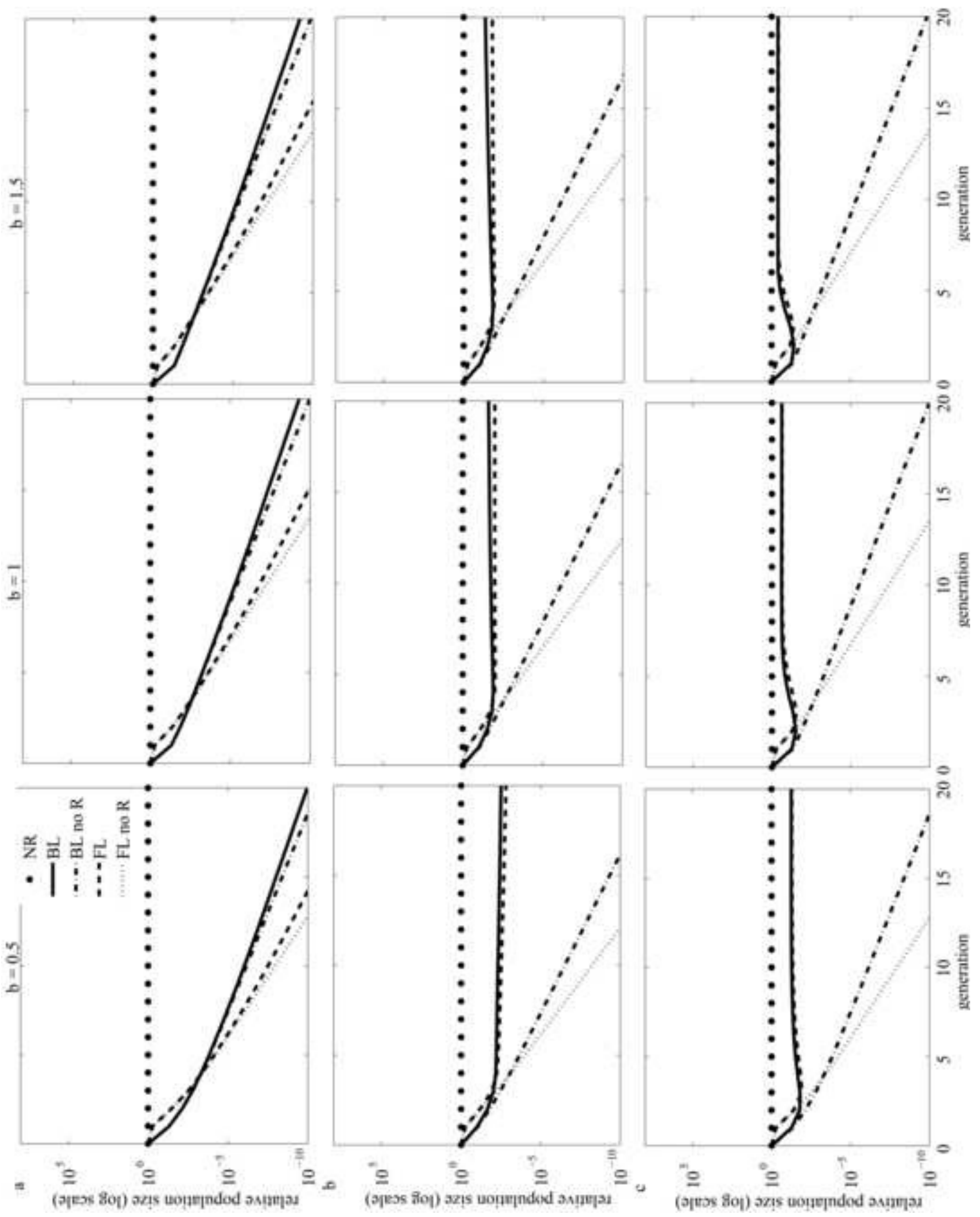

